Supporting Information

\title{
The Spirastrellolides: Construction of the Southern C(1)-C(25) Fragment Exploiting Anion Relay Chemistry
}

Amos B. Smith, III* and Dae-Shik Kim

Department of Chemistry, Laboratory for Research on the Structure of Matter, and Monell

Chemical Senses Center, University of Pennsylvania, Philadelphia, PA 19104, U.S.A.

Table of Contents:

-General Methods and Experimental Procedures

S2-S36

${ }^{1} \mathrm{H}$ and ${ }^{13} \mathrm{C}$ NMR Spectra

S37-S104 
Experimental

I. Materials and Methods

Except as otherwise indicated, reactions were carried out under an argon atmosphere in flame- or oven-dried glassware. In aqueous work-up, all organic solutions were dried over sodium sulfate or magnesium sulfate, and filtered prior to rotary evaporation at water aspirator pressure. All solvents were freshly distilled and dried by standard techniques just before use. Diethyl ether and THF were distilled from sodium/benzophenone. After storing over 4A molecular sieves for several days, HPLC grade dichloromethane was used without further purification. KO-t-Bu (1.0 M in THF) and $n$-BuLi were purchased from Aldrich. Reactions were monitored by thin layer chromatography (TLC) with 0.25-mm E. Merck pre-coated silica gel plates (Kieselgel $60 \mathrm{~F}_{254}$, Merck). Spots were detected by viewing under a UV light, putting into an iodine chamber, or colorizing with charring after dipping in an anisaldehyde solution composed of acetic acid, sulfuric acid, and $\mathrm{MeOH}$. Silica gel for flash chromatography (particle size 0.040-0.063 mm) was supplied by Silicycle and Sorbent technologies. Yields refer to chromatographically and spectroscopically pure compounds unless otherwise noted. ${ }^{1} \mathrm{H}$ and ${ }^{13} \mathrm{C}$ spectra were recorded on a Bruker AM-500 spectrometer. Chemical shifts are reported as $\delta$ values relative to internal chloroform ( $\delta 7.26$ for ${ }^{1} \mathrm{H}$ and $\delta 77.0$ for ${ }^{13} \mathrm{C}$ ) or benzene $\left(\delta 7.16\right.$ for ${ }^{1} \mathrm{H}$ and $\delta 128.39$ for $\left.{ }^{13} \mathrm{C}\right)$. IR spectra were measured as neat oils on a Perkin-Elmer Model 1600 FTIR. Optical rotations were measured on a Jasco polarimeter. High resolution mass spectra were obtained at the University of Pennsylvania Mass Spectrometry Service Center.

II. Procedure 


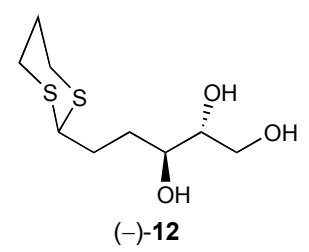

Triol (-)-12. To a solution of (-)-tri-O-acetyl-D-glucal $(10 \mathrm{~g}, 36.7 \mathrm{mmol})$ in $\mathrm{CH}_{2} \mathrm{Cl}_{2}(60 \mathrm{~mL})$ at $0{ }^{\circ} \mathrm{C}$ was added $\mathrm{MeOH}\left(6 \mathrm{~mL}, 150 \mathrm{mmol}, 6\right.$ equiv) followed by $\mathrm{BF}_{3} \cdot \mathrm{OEt}_{2}(6.0 \mathrm{~mL}, 47 \mathrm{mmol}, 1.3$ equiv). The resulting solution was stirred for $20 \mathrm{~min}$ at $0{ }^{\circ} \mathrm{C}$ and for $40 \mathrm{~min}$ at ambient temperature and the reaction was then quenched with a saturated aqueous $\mathrm{NaHCO}_{3}$ solution $(60 \mathrm{~mL})$. The mixture was diluted with EtOAc $(400 \mathrm{~mL})$ and the organic layer was washed with a saturated aqueous $\mathrm{NaHCO}_{3}$ solution $\left(150 \mathrm{~mL}\right.$ ) and brine $(150 \mathrm{~mL})$, dried over $\mathrm{Na}_{2} \mathrm{SO}_{4}$, filtered and concentrated in vacuo. The crude methyl glycoside was dissolved in EtOAc/ethanol $(50 / 50 \mathrm{~mL})$ at ambient temperature and $10 \% \mathrm{Pd} / \mathrm{C}(1.2 \mathrm{~g})$ was added. The flask was charged with hydrogen (balloon) and the mixture was stirred overnight under hydrogen atmosphere. The catalyst was removed by filtration through a pad of Celite and the filtrate was concentrated in vacuo. The resulting residue was dissolved in $\mathrm{MeOH}(100 \mathrm{~mL})$ at ambient temperature and $\mathrm{NaOMe}(0.53 \mathrm{~g}$, $9.8 \mathrm{mmol}, 0.27$ equiv) was added. After $3 \mathrm{~h}$ at ambient temperature, the reaction was quenched with Amberlyst-15. The resin was removed by filtration and the solvent was removed in vacuo. The resulting crude diol product was dissolved in $\mathrm{CHCl}_{3}(15 \mathrm{~mL})$ and 1,3-propanethiol $(5.5 \mathrm{~mL}, 55$ mol, 1.5 equiv) was added. After the resulting solution was cooled to $0{ }^{\circ} \mathrm{C}$, concentrated $\mathrm{HCl}(12$ $\mathrm{N}, 10 \mathrm{~mL}$ ) was added slowly. After being stirred for $30 \mathrm{~min}$ at ambient temperature, the mixture was poured into a mixture of $4 \mathrm{M} \mathrm{NaOH}(30 \mathrm{~mL})$ and saturated $\mathrm{NaHCO}_{3}(50 \mathrm{~mL})$ at $0{ }^{\circ} \mathrm{C}$ slowly. The resulting mixture was stirred for $10 \mathrm{~min}$ at $0{ }^{\circ} \mathrm{C}$ and extracted with EtOAc (100 mL X 10). The organic layer was dried over $\mathrm{MgSO}_{4}$, filtered and concentrated in vacuo to give a white solid. Recrystalization (2 times) of the product from ethyl acetate provided triol (-)-12 (6.65 g, 27.9 mmol, $76 \%$ yield over 4 steps) as a white solid: $R_{f} 0.15$ (ethyl acetate); $[\alpha]^{20}{ }_{D}-20.5$ (c 1.00, $\mathrm{CH}_{3} \mathrm{OH}$ ); m.p. = 98-99 ${ }^{\circ} \mathrm{C}$; IR (film) 3435 (br s), 3278 (br s), 2924 (m), 1448 (m), 1412 (s), 1280 (m), 1111 (s), 1006 (s) $\mathrm{cm}^{-1} ;{ }^{1} \mathrm{H}$ NMR $\left(500 \mathrm{MHz}, \mathrm{CDCl}_{3}\right) \delta 4.09$ (app t, $\left.J=6.9 \mathrm{~Hz}, 1 \mathrm{H}\right), 3.83$ (dd, $J$ $=11.3$ and $5.5 \mathrm{~Hz}, 1 \mathrm{H}), 3.62-3.59(\mathrm{~m}, 1 \mathrm{H}), 2.91-2.82(\mathrm{~m}, 4 \mathrm{H}), 2.17-2.10(\mathrm{~m}, 4 \mathrm{H})$, 2.10-2.02 $(\mathrm{m}$, 
1H), $1.92-1.81(\mathrm{~m}, 2 \mathrm{H}), 1.80-1.76(\mathrm{~m}, 1 \mathrm{H}), 1.71-1.63(\mathrm{~m}, 1 \mathrm{H}) ;{ }^{13} \mathrm{C} \mathrm{NMR}\left(125 \mathrm{MHz}, \mathrm{CDCl}_{3}\right) \delta 73.5$, 73.3, 63.3, 47.3, 32.0, 30.4(2C), 29.9, 25.9; high resolution mass spectrum (ESI) m/z 239.0764 $\left[(\mathrm{M}+\mathrm{H})^{+} ;\right.$calcd for $\left.\mathrm{C}_{9} \mathrm{H}_{20} \mathrm{O}_{3} \mathrm{~S}_{2}: 239.0776\right]$.

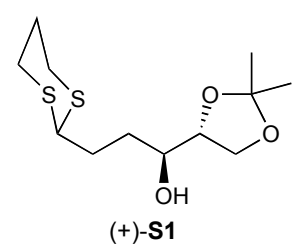

Acetonide (+)-S1. To a slurry of triol $(+)-12(6.64 \mathrm{~g}, 27.9 \mathrm{mmol})$ in acetone $(110 \mathrm{~mL})$ at $-78{ }^{\circ} \mathrm{C}$ was added 2.2-dimethoxypropane $\left(11 \mathrm{~mL}, 89 \mathrm{mmol}, 3.2\right.$ equiv) and then $p-\mathrm{TsOH} \bullet \mathrm{H}_{2} \mathrm{O}(0.50 \mathrm{~g}$, $0.028 \mathrm{mmol}, 0.1$ equiv). The resulting mixture was warmed to $-25^{\circ} \mathrm{C}$ over $2 \mathrm{~h}$. At that time, the reaction mixture became a clear solution. Then $0.5 \mathrm{~mL}$ of triethylamine was added and the solvent was removed in vacuo. Flash chromatography on silica gel, using ethyl acetate-hexanes $(1: 2 \rightarrow 1: 1)$ as eluant, provided acetonide (+)-S1 $(5.44 \mathrm{~g}, 19.5 \mathrm{mmol}, 70 \%$ yield) as a colorless oil: $R_{f} 0.20$ (hexanes/ethyl acetate 2/1); $[\alpha]^{20}{ }_{D}+4.4\left(c\right.$ 1.0, $\mathrm{CHCl}_{3}$ ); IR (film) 3465 (br s), 2985 (s), 2898 (s), 1636 (w), 1371 (s), 1213 (s), 1066 (s), 856 (s), 908 (s) cm ${ }^{-1} ;{ }^{1} \mathrm{H}$ NMR (500 MHz, CDCl ${ }_{3}$ ) $\delta 4.02(\operatorname{app~t}, J=6.9 \mathrm{~Hz}, 1 \mathrm{H}), 3.98-3.92(\mathrm{~m}, 2 \mathrm{H}), 3.84(\mathrm{dd}, J=7.4$ and $6.8 \mathrm{~Hz}, 1 \mathrm{H}), 3.70($ app dt, $J=9.5$ and $3.8 \mathrm{~Hz}, 1 \mathrm{H}), 2.85-2.76(\mathrm{~m}, 4 \mathrm{H}), 2.26(\mathrm{~s}, 1 \mathrm{H}), 2.09-2.04(\mathrm{~m}, 1 \mathrm{H}), 2.03-1.95(\mathrm{~m}, 1 \mathrm{H})$, 1.85-1.75 (m, 2H), 1.69 (dddd, $J=13.7,9.7,5.8$ and $3.3 \mathrm{~Hz}, 1 \mathrm{H}), 1.50-1.42(\mathrm{~m}, 1 \mathrm{H}), 1.37(\mathrm{~s}, 3 \mathrm{H})$, 1.30 (s, 3H); ${ }^{13} \mathrm{C}$ NMR $\left(125 \mathrm{MHz}, \mathrm{CDCl}_{3}\right) \delta 108.9,78.4,70.3,64.6,47.2,31.6,30.2$ (2C), 29.6, 26.4, 25.8, 25.1; high resolution mass spectrum $(\mathrm{ESI}) \mathrm{m} / \mathrm{z} 301.0918\left[(\mathrm{M}+\mathrm{Na})^{+}\right.$; calcd for $\mathrm{C}_{12} \mathrm{H}_{22} \mathrm{O}_{3} \mathrm{NaS}_{2}$ : 301.0908].

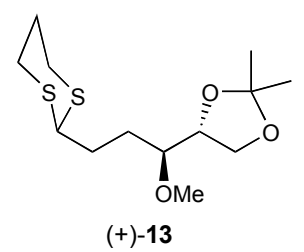

Dithiane (+)-13. To a solution of (+)-S1 $(5.40 \mathrm{~g}, 19.4 \mathrm{mmol})$ in THF $(60 \mathrm{~mL})$ at $0{ }^{\circ} \mathrm{C}$ was added $\mathrm{NaH}$ (60\% in oil, $1.16 \mathrm{~g}, 29 \mathrm{mmol}, 1.5$ equiv). After being stirred for $2 \mathrm{~h}$ at ambient temperature, 
the mixture was cooled to $0{ }^{\circ} \mathrm{C}$ and treated with dimethyl sulfate $(3.7 \mathrm{~mL}, 39 \mathrm{mmol}, 2.0$ equiv). The resulting reaction mixture was warmed to ambient temperature and stirred for $2.5 \mathrm{~h}$. A saturated aqueous $\mathrm{NH}_{4} \mathrm{Cl}$ solution $(100 \mathrm{~mL})$ was then added and the mixture was extracted with $\mathrm{Et}_{2} \mathrm{O}(100 \mathrm{~mL} \times 3)$. The combined organic layers were washed with brine $(100 \mathrm{~mL})$, dried over $\mathrm{MgSO}_{4}$, filtered and concentrated in vacuo. Flash chromatography on silica gel, using ethyl acetate-hexanes (1:4) as eluant, provided dithiane (+)-13 (4.78 g, $16.3 \mathrm{mmol}, 84 \%$ yield) as a colorless oil: $\mathrm{R}_{\mathrm{f}} 0.50$ (hexanes/ethyl acetate 4/1); $[\alpha]^{20}{ }_{\mathrm{D}}+7.4\left(c \quad 0.32, \mathrm{CHCl}_{3}\right)$; IR (film) 2984 (s), 2933 (s), 2825(m), 1455 (m), 1423 (m), 1370 (s), 1213 (s), 1105 (s), 908 (m), 851 (s) cm ${ }^{-1} ;{ }^{1} \mathrm{H}$ NMR (500 MHz, $\left.\mathrm{CDCl}_{3}\right) \delta$ 4.04-3.99 (m, 3H), 3.86-3.81 (m, 1H), $3.40(\mathrm{~s}, 3 \mathrm{H}), 3.26-3.23(\mathrm{~m}, 1 \mathrm{H})$, 2.89-2.80 (m, 4H), 2.13-2.08 (m, 1H), 1.97-1.79 (m, 4H), 1.70-1.61 (m, 1H), $1.40(\mathrm{~s}, 3 \mathrm{H}), 1.33(\mathrm{~s}$, $3 \mathrm{H}) ;{ }^{13} \mathrm{C}$ NMR $\left(125 \mathrm{MHz}, \mathrm{CDCl}_{3}\right) \delta 109.1,80.8,77.1,66.5,58.4,47.6,30.6,30.33,30.32 .27 .5$, 26.5, 26.0, 25.3; high resolution mass spectrum $(\mathrm{ESI}) \mathrm{m} / \mathrm{z} 315.1065\left[(\mathrm{M}+\mathrm{Na})^{+}\right.$; calcd for $\left.\mathrm{C}_{13} \mathrm{H}_{24} \mathrm{O}_{3} \mathrm{NaS}_{2}: 315.1065\right]$

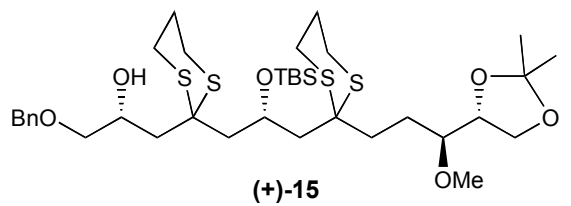

Alcohol (+)-15. To a solution of dithiane $(+)-13(3.00 \mathrm{~g}, 10.3 \mathrm{mmol}, 1.0$ equiv) in THF $(30 \mathrm{~mL})$ at $-78{ }^{\circ} \mathrm{C}$ was added KO-t-Bu (1.0 M in THF, $10.3 \mathrm{~mL}, 10.3 \mathrm{mmol}, 1.0$ equiv) and then $n$-BuLi (2.2 M in hexane, $4.66 \mathrm{~mL}, 10.3 \mathrm{mmol}, 1.0$ equiv). The resulting solution was stirred for $30 \mathrm{~min}$ at $-78{ }^{\circ} \mathrm{C}$ and a solution of epoxide (-)-11 $(2.98 \mathrm{~g}, 10.3 \mathrm{mmol})$ in THF $(10 \mathrm{~mL})$ was added. After $2 \mathrm{~h}$ at -78 ${ }^{\circ} \mathrm{C}$, a solution of epoxide (-)-10 (2.53 g, $15.4 \mathrm{mmol}, 1.5$ equiv) in THF (3 mL) was added via cannula and the resulting solution was stirred for $30 \mathrm{~min}$ at $-78{ }^{\circ} \mathrm{C}$ and for an additional $20 \mathrm{~min}$ at $0{ }^{\circ} \mathrm{C}$. The reaction was quenched with a saturated aqueous $\mathrm{NH}_{4} \mathrm{Cl}$ solution $(50 \mathrm{~mL})$. The resulting mixture was then extracted with $\mathrm{Et}_{2} \mathrm{O}(50 \mathrm{~mL} \times 3)$. The combined organic layers were washed with brine $(50 \mathrm{~mL})$, dried over $\mathrm{MgSO}_{4}$, filtered and concentrated in vacuo. Flash chromatography on silica gel, using ethyl acetate-hexanes (1:3) as eluant, provided (+)-15 (5.89 g, $7.88 \mathrm{mmol}, 77 \%$ yield) as a pale yellow oil: $R_{f} 0.45$ (hexanes/ethyl acetate $\left.2 / 1\right) ;[\alpha]^{20}+3.3(c$ 1.0, $\mathrm{CHCl}_{3}$ ); IR (film) 3473 (br m), 3032 (w), 2929 (s), 2855 (s), 1689 (w), 1454 (m), 1369 (m), 
$1256(\mathrm{~m}), 1092$ (s), $836(\mathrm{~s}) \mathrm{cm}^{-1} ;{ }^{1} \mathrm{H}$ NMR $\left(500 \mathrm{MHz}, \mathrm{CDCl}_{3}\right) \delta$ 7.34-7.32 (m, 4H), 7.29-7.26 (m, 1H), $4.57(\mathrm{~s}, 2 \mathrm{H}), 4.56-4.52(\mathrm{~m}, 1 \mathrm{H}), 4.33-4.30(\mathrm{~m}, 1 \mathrm{H}), 4.06-4.02(\mathrm{~m}, 2 \mathrm{H}), 3.89-3.85(\mathrm{~m}, 1 \mathrm{H})$, $3.47(\mathrm{dd}, J=9.5$ and $4.6 \mathrm{~Hz}, 1 \mathrm{H}), 3.43(\mathrm{dd}, J=9.5$ and $6.6 \mathrm{~Hz}, 1 \mathrm{H}), 3.42(\mathrm{~s}, 3 \mathrm{H}), 3.28(\operatorname{app~q}, J=$ $4.9 \mathrm{~Hz}, 1 \mathrm{H}), 3.05(\mathrm{app} d t, J=13.9$ and $5.8 \mathrm{~Hz}, 1 \mathrm{H}), 2.96-2.74(\mathrm{~m}, 7 \mathrm{H}), 2.45(\mathrm{dd}, J=15.1$ and 6.7 $\mathrm{Hz}, 1 \mathrm{H}), 2.39(\mathrm{dd}, J=15.4$ and $8.2 \mathrm{~Hz}, 1 \mathrm{H}), 2.17-2.11(\mathrm{~m}, 2 \mathrm{H}), 2.06(\mathrm{dd}, J=15.4$ and $2.2 \mathrm{~Hz}$, 1H), $1.99(\mathrm{dd}, J=15.1$ and $6.7 \mathrm{~Hz}, 1 \mathrm{H}), 1.97-1.90(\mathrm{~m}, 6 \mathrm{H}), 1.86-1.79(\mathrm{~m}, 1 \mathrm{H}), 1.76-1.70(\mathrm{~m}, 1 \mathrm{H})$, $1.41(\mathrm{~s}, 3 \mathrm{H}), 1.34(\mathrm{~s}, 3 \mathrm{H}), 0.88(\mathrm{~s}, 9 \mathrm{H}), 0.17$ (s, 3H), $0.16(\mathrm{~s}, 3 \mathrm{H}) ;{ }^{13} \mathrm{C}$ NMR $\left(125 \mathrm{MHz}, \mathrm{CDCl}_{3}\right)$ $\delta 138.2,128.3,127.7,127.6,109.0,81.3,77.3,74.5,73.3,68.5,67.9,66.5,58.4,52.5,51.7$, $48.4,47.8,44.2,35.3,26.8,26.6,26.4,26.22,26.15,25.7,25.3,25.2,24.9,18.0,-3.4$; high resolution mass spectrum (ESI) m/z 769.3061 [(M+Na) ${ }^{+}$; calcd for $\mathrm{C}_{36} \mathrm{H}_{62} \mathrm{O}_{6} \mathrm{NaSiS}_{4}:$ 769.3096].

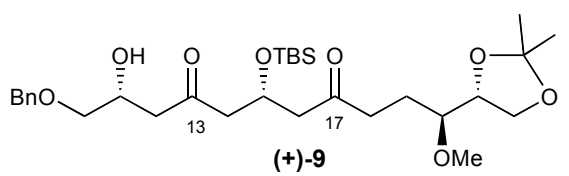

Ketone (+)-9. To a solution of dithiane (+)-15 $(7.27 \mathrm{~g}, 9.73 \mathrm{mmol})$ in $\mathrm{THF} / \mathrm{H}_{2} \mathrm{O}(100 / 20 \mathrm{~mL})$ at 0 ${ }^{\circ} \mathrm{C}$ was added $\mathrm{CaCO}_{3}\left(5.3 \mathrm{~g}, 53 \mathrm{mmol}, 5.4\right.$ equiv) and then $\mathrm{Hg}\left(\mathrm{ClO}_{4}\right)_{2} \bullet 3 \mathrm{H}_{2} \mathrm{O}(17 \mathrm{~g}, 43$ mmol. 4.4 equiv). After being stirred for $5 \mathrm{~min}$ at $0{ }^{\circ} \mathrm{C}$, the reaction mixture was filtered through a short pad of Celite, followed by a rinse with $\mathrm{Et}_{2} \mathrm{O}$. The combined filtrates were concentrated in vacuo. Flash chromatography on silica gel, using ethyl acetate-hexanes (1:1.5) as eluant, provided ketone (+)-9 (4.14 g, $7.30 \mathrm{mmol}, 75 \%$ yield) as a pale yellow oil: $R_{f} 0.30$ (hexanes/ethyl acetate 1/1); $[\alpha]^{20}{ }_{\mathrm{D}}+3.39\left(\mathrm{c}\right.$ 0.53, $\left.\mathrm{CHCl}_{3}\right) ; \mathrm{IR}$ (film) 3479 (br s), 3064 (w), 3031 (m), 2930 (s), 2857 (s), 1713 (s), 1496 (m), 1455 (s), 1371 (s), 1255 (s), 1090 (s), 838 (s), 699 (s) cm ${ }^{-1}$; ${ }^{1} \mathrm{H}$ NMR (500 $\left.\mathrm{MHz} \mathrm{CDCl}_{3}\right) \delta$ 7.37-7.28 (m, 5H), 4.60-4.56 (m, 1H), $4.55(\mathrm{~d}, J=3.2 \mathrm{~Hz}, 2 \mathrm{H}), 4.28-4.24(\mathrm{~m}, 1 \mathrm{H})$, 4.05-3.98 (m, 2H), $3.84(\mathrm{dd}, J=7.6$ and $5.6 \mathrm{~Hz}, 1 \mathrm{H}), 3.48(\mathrm{dd}, J=9.6$ and $4.6 \mathrm{~Hz}, 1 \mathrm{H}), 3.44(\mathrm{dd}$, $J=9.6$ and $6.0 \mathrm{~Hz}, 1 \mathrm{H}), 3.37(\mathrm{~s}, 3 \mathrm{H}), 3.22(\mathrm{ddd}, J=6.4,6.4$ and $4.2 \mathrm{~Hz}, 1 \mathrm{H}), 2.71-2.48(\mathrm{~m}, 8 \mathrm{H})$, 1.92-1.85 (m, 1H), 1.74-168. (m, 1H), $1.40(\mathrm{~s}, 3 \mathrm{H}), 1.33(\mathrm{~s}, 3 \mathrm{H}), 0.84(\mathrm{~s}, 9 \mathrm{H}), 0.06(\mathrm{~s}, 6 \mathrm{H}) ;{ }^{13} \mathrm{C}$ NMR $\left(125 \mathrm{MHz}, \mathrm{CDCl}_{3}\right) \delta 208.7$ (2C), 137.9, 128.3, 127.7, 109.1, 80.4, 76.9, 73.3, 73.2, 66.6, 
$66.5,65.1,58.2,50.4,49.6,47.3,39.2,26.5,25.7,25.2,23.9,17.8,-4.9$; high resolution mass spectrum (ESI) m/z 589.3148 [(M+Na) $)^{+}$; calcd for $\mathrm{C}_{30} \mathrm{H}_{50} \mathrm{O}_{8} \mathrm{NaSi}:$ 589.3173].

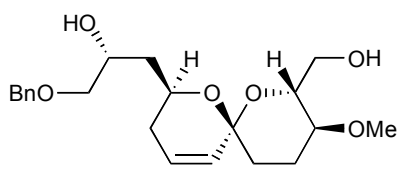

$(+)-17$

Sprioketal (+)-17. To a solution of $\mathrm{Me}_{4} \mathrm{NHB}(\mathrm{OAc})_{3}\left(14 \mathrm{~g}, 53 \mathrm{mmol}, 6.0\right.$ equiv) in $\mathrm{CH}_{3} \mathrm{CN}(90 \mathrm{~mL})$ at ambient temperature was added $\mathrm{AcOH}(30 \mathrm{~mL})$. After being stirred for 40 min at ambient temperature, the mixture was cooled to $-35^{\circ} \mathrm{C}$, and a solution of ketone $(+)-9(4.80 \mathrm{~g}, 8.47 \mathrm{mmol})$ in $\mathrm{CH}_{3} \mathrm{CN}(20 \mathrm{~mL})$ was added slowly. The reaction mixture was stirred for 4 days at $-35{ }^{\circ} \mathrm{C}$, and it was then poured into a mixture of saturated Rochelle's salt solution $(100 \mathrm{~mL})$ and water $(150 \mathrm{~mL})$ at $0{ }^{\circ} \mathrm{C}$, and rinsed with $\mathrm{Et}_{2} \mathrm{O}(50 \mathrm{~mL})$. After 30 min stirring at ambient temperature, the organic layer was separated. The aqueous layer was extracted with diethyl ether ( $200 \mathrm{~mL} X 2)$. The combined organic layers were washed with a saturated $\mathrm{NaHCO}_{3}$ solution $(200 \mathrm{~mL})$ and brine (100 mL), dried over $\mathrm{MgSO}_{4}$, filtered and concentrated in vacuo. The residue was dissolved in acetone $(200 \mathrm{~mL})$ and $\mathrm{pTsOH} \bullet \mathrm{H}_{2} \mathrm{O}(0.15 \mathrm{~g}, 0.79 \mathrm{mmol}, 0.09$ equiv) was added at ambient temperature. After $8 \mathrm{~min}$, the reaction was quenched with a saturated $\mathrm{NaHCO}_{3}$ solution $(50 \mathrm{~mL})$. The resulting mixture was diluted with water $(50 \mathrm{~mL})$ and extracted with $\mathrm{Et}_{2} \mathrm{O}(150 \mathrm{~mL} X 3)$. The combined organic layers were washed brine $(50 \mathrm{~mL})$, dried over $\mathrm{MgSO}_{4}$, filtered and concentrated in vacuo. The resulting residue was dissolved in $\mathrm{CH}_{2} \mathrm{Cl}_{2}(150 \mathrm{~mL})$ and treated with a $3.5 \%$ aqueous $\mathrm{HClO}_{4}(75 \mathrm{~mL})$ at ambient temperature. After $36 \mathrm{~h}$ stirring at ambient temperature, the organic layer was separated, and the aqueous layer was extracted with $\mathrm{CH}_{2} \mathrm{Cl}_{2}$ $(50 \mathrm{~mL} \times 2)$. The combined organic layers were washed with a saturated $\mathrm{NaHCO}_{3}$ solution (50 $\mathrm{mL}$ ) and brine $(50 \mathrm{~mL})$, dried over $\mathrm{MgSO}_{4}$, filtered and concentrated in vacuo. Flash chromatography on silica gel, using ethyl acetate as eluant, provided (+)-17 $(2.0 \mathrm{~g}, 5.28 \mathrm{mmol}$, $63 \%$ yield over 3 steps) as a pale yellow oil: $R_{f} 0.50$ (ethyl acetate); $[\alpha]^{20}{ }_{D}+20.3\left(c 1.00, \mathrm{CHCl}_{3}\right)$; IR (film) 3435 (br s), 3034 (w), 2937 (s), 1713 (w), 1657 (w). 1453 (m), 1365 (m), 1237 (m), 1097 (s), 699 (s) cm ${ }^{-1} ;{ }^{1} \mathrm{H} \mathrm{NMR}\left(500 \mathrm{MHz}, \mathrm{CDCl}_{3}\right) \delta$ 7.36-7.29 (m, 5H), 5.95 (ddd, $J=10.0,5.3$ and 2.3 
$\mathrm{Hz}, 1 \mathrm{H}$ ), 5.59 (ddd, $J=10.0,1.3$ and $1.2 \mathrm{~Hz}, 1 \mathrm{H}), 4.57$ (s, 2H), 4.27-4.23 (m, 1H), 4.21-4.18 (m, 1H), $3.84(\mathrm{dd}, J=11.2$ and $3.2 \mathrm{~Hz}, 1 \mathrm{H}), 3.77(\mathrm{ddd}, J=9.2,5.6$ and $3.2 \mathrm{~Hz}, 1 \mathrm{H}), 3.67(\mathrm{dd}, J=$ 11.2 and $5.6 \mathrm{~Hz}, 1 \mathrm{H}), 3.53(\mathrm{dd}, J=9.4$ and $3.6 \mathrm{~Hz}, 1 \mathrm{H}), 3.37(\mathrm{dd}, J=9.4$ and $7.1 \mathrm{~Hz}, 1 \mathrm{H}), 3.36$ (s, 3H), $3.16(\mathrm{ddd}, J=10.1,10.1$ and $4.4 \mathrm{~Hz}, 1 \mathrm{H}), 2.08-2.02(\mathrm{~m}, 1 \mathrm{H}), 2.00-1.94(\mathrm{~m}, 2 \mathrm{H}), 1.83$ (ddd, $J=12.9 .3 .7$ and $3.2 \mathrm{~Hz}, 1 \mathrm{H}), 1.78-1.71(\mathrm{~m}, 1 \mathrm{H}), 1.69-1.60(\mathrm{~m}, 3 \mathrm{H}) ;{ }^{13} \mathrm{C}$ NMR $(125 \mathrm{MHz}$, $\left.\mathrm{CDCl}_{3}\right) \delta 138.0,129.3,128.5,128.3,127.7,127.6,94.1,75.5,74.8,73.3,73.2,66.4,63.9,62.9$, 56.2, 38.6, 33.8, 30.8, 23.9; high resolution mass spectrum (ESI) m/z $401.1951\left[(\mathrm{M}+\mathrm{Na})^{+}\right.$; calcd for $\mathrm{C}_{21} \mathrm{H}_{30} \mathrm{O}_{6} \mathrm{Na:}$ 401.1940].

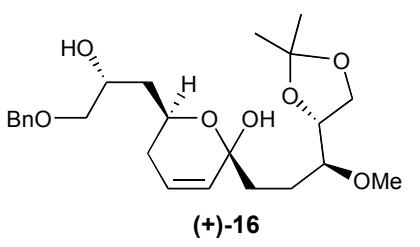

Hemiketal (+)-16: $\mathrm{R}_{\mathrm{f}} 0.75$ (hexanes/ethyl acetate 1/1); $[\alpha]^{20}{ }_{\mathrm{D}}+30.5\left(\mathrm{c} 1.00, \mathrm{CHCl}_{3}\right) ; \mathrm{IR}(\mathrm{film}) 3501$ (br m), 3063 (w), 3032 (m), 2934 (s), 1714 (w), 1656 (w). 1496 (m), 1454 (s), 1369 (s), 1213 (s), 1094 (s), 853 (s), 738 (s) cm ${ }^{-1} ;{ }^{1} \mathrm{H}$ NMR (500 MHz, $\left.\mathrm{CDCl}_{3}\right) \delta$ 7.35-7.32 (m, 4H), 7.32-7.26 (m, 1H), $6.15(\mathrm{ddd}, J=10.0,4.4$ and $3.5 \mathrm{~Hz}, 1 \mathrm{H}), 5.57(\mathrm{ddd}, J=10.0,2.0$ and $2.0 \mathrm{~Hz}, 1 \mathrm{H}), 4.58\left(\mathrm{~d}, J_{\mathrm{AB}}=\right.$ 12.3, $1 \mathrm{H}), 4.55\left(\mathrm{~d}, \mathrm{~J}_{\mathrm{AB}}=12.3,1 \mathrm{H}\right), 4.39(\operatorname{app~t}, J=6.5 \mathrm{~Hz}, 1 \mathrm{H}), 3.89-3.85(\mathrm{~m}, 1 \mathrm{H}), 4.33-4.28(\mathrm{~m}$, 1H), 4.04-3.99 (m, 1H), $3.51(\mathrm{dd}, J=10.0$ and $5.7 \mathrm{~Hz}, 1 \mathrm{H}), 3.41(\mathrm{~s}, 3 \mathrm{H}), 3.38(\mathrm{dd}, J=10.0$ and 4.8 Hz, 1H), 3.31-3.27 (m, 1H), 2.65-2.60 (m, 1H), 1.96-1.84 (m, 3H), 1.76-1.61 (m, 4H), 1.43$1.39(\mathrm{~m}, 1 \mathrm{H}), 1.40$ (s, 3H), 1.33 (s, 3H); ${ }^{13} \mathrm{C}$ NMR $\left(125 \mathrm{MHz}, \mathrm{CDCl}_{3}\right) \delta 138.3,129.5,128.3,127.7$, $127.6,127.0,108.9,94.3,81.1,77.7,73.7,73.4,66.9,66.4,66.3,58.5,35.0,33.6,29.5,26.5$, 25.4, 23.9; high resolution mass spectrum (ESI) $\mathrm{m} / \mathrm{z} 441.2268\left[\left(\mathrm{M}-\mathrm{H}_{2} \mathrm{O}+\mathrm{Na}\right)^{+}\right.$; calcd for $\left.\mathrm{C}_{24} \mathrm{H}_{34} \mathrm{O}_{6} \mathrm{Na}: 441.2253\right]$.

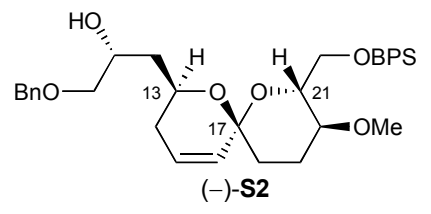


BPS ether (-)-S2. To a solution of (+)-17 $(0.950 \mathrm{~g}, 2.51 \mathrm{mmol})$ in $\mathrm{CH}_{2} \mathrm{Cl}_{2}(15 \mathrm{~mL})$ at $0{ }^{\circ} \mathrm{C}$ were added imidazole (0.256 g, $3.76 \mathrm{mmol}, 1.5$ equiv), DMAP (0.030 g, $0.25 \mathrm{mmol}, 0.10$ equiv), and $\mathrm{BPSCl}\left(0.690 \mathrm{~mL}, 2.64 \mathrm{mmol}, 1.05\right.$ equiv). After being stirred for $30 \mathrm{~min}$ at $0{ }^{\circ} \mathrm{C}$, the mixture was poured into a saturated aqueous $\mathrm{NH}_{4} \mathrm{Cl}$ solution $(30 \mathrm{~mL})$ and the resulting mixture was extracted with $\mathrm{CH}_{2} \mathrm{Cl}_{2}(50 \mathrm{~mL} \times 2)$. The combined organic layers were washed with brine $(50 \mathrm{~mL})$, dried over $\mathrm{MgSO}_{4}$, filtered and concentrated in vacuo. Flash chromatography on silica gel, using ethyl acetate-hexanes $(1: 7 \rightarrow 1: 4)$ as eluant, provided $(-)$-S2 $(1.30 \mathrm{~g}, 2.11 \mathrm{mmol}, 84 \%$ yield $)$ as a colorless oil: $\mathrm{R}_{\mathrm{f}} 0.40$ (hexanes/ethyl acetate 3/1); $[\alpha]^{20}{ }_{\mathrm{D}}-20.1$ (c 2.14, $\mathrm{CHCl}_{3}$ ); IR (film) 3458 (br s), 3044 (m), 2931 (s), 2856 (s), 1654 (w). 1589 (w), 1428 (m), 1235 (m), 1111 (s), 1016 (s), 701 (s) $\mathrm{cm}^{-1} ;{ }^{1} \mathrm{H}$ NMR $\left(500 \mathrm{MHz}, \mathrm{CDCl}_{3}\right) \delta$ 7.78-7.74 (m, 4H), 7.41-7.31 (m, 10H), 7.29-7.26 (m, 1H), 5.94 (ddd, $J=9.9,5.5$ and $2.1 \mathrm{~Hz}, 1 \mathrm{H}$ ), $5.62($ app dt, $J=10.0$ and $1.1 \mathrm{~Hz}, 1 \mathrm{H}), 4.54$ (s, 2H), 4.24 (ddd, $J=11.8,7.8,3.9$ and $3.9 \mathrm{~Hz}, 1 \mathrm{H}), 4.17-4.12(\mathrm{~m}, 1 \mathrm{H}), 3.91(\mathrm{dd}, J=11.0$ and $4.2 \mathrm{~Hz}, 1 \mathrm{H}), 3.85$ (dd, $J=11.0$ and $1.5 \mathrm{~Hz}, 1 \mathrm{H}$ ), 3.72 (ddd, $J=9.6,4.1$ and $1.7 \mathrm{~Hz}, 1 \mathrm{H}$ ), 3.50 (dd, $J=9.4$ and 3.7 $\mathrm{Hz}, 1 \mathrm{H}), 3.37(\mathrm{~s}, 3 \mathrm{H}), 3.37-3.33(\mathrm{~m}, 2 \mathrm{H}), 2.09-2.04(\mathrm{~m}, 1 \mathrm{H}), 2.01(\mathrm{app} d t, J=10.6$ and $2.4 \mathrm{~Hz}$, 1H), 1.98-1.93 (m, 1H), $1.83($ app dt, $J=12.7$ and $3.2 \mathrm{~Hz}, 1 \mathrm{H}), 1.78-1.73(\mathrm{~m}, 1 \mathrm{H}), 1.70-1.61(\mathrm{~m}$, 3H), 1.05 (s, 9H); ${ }^{13} \mathrm{C}$ NMR $\left(125 \mathrm{MHz}, \mathrm{CDCl}_{3}\right) \delta 138.1,135.9,135.8,134.2,133.9,130.0,129.35$, $129.34,128.4,127.8,127.7,127.42,127.39,94.0,74.9,74.3,73.9,67.1,64.3,63.8,56.5,38.6$, 34.0, 30.7, 26.8, 24.0, 19.4; high resolution mass spectrum (ESI) $\mathrm{m} / \mathrm{z} 639.3110\left[(\mathrm{M}+\mathrm{Na})^{+}\right.$; calcd for $\mathrm{C}_{37} \mathrm{H}_{48} \mathrm{O}_{8} \mathrm{NaSi}$ : 639.3118].

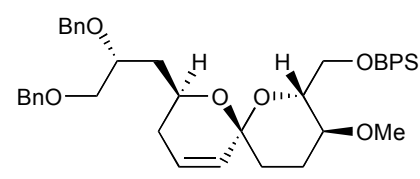

(+)-S3

Benzyl ether (+)-S3. To a solution of $(-)-S 2(1.30 \mathrm{~g}, 2.11 \mathrm{mmol})$ in DMF $(10 \mathrm{~mL})$ in $0{ }^{\circ} \mathrm{C}$ was added $\mathrm{NaH}(0.127 \mathrm{~g}, 60 \%$ in mineral oil, $3.2 \mathrm{mmol}, 1.5$ equiv). The resulting mixture was warmed to ambient temperature and stirred for 15 min before it was cooled to $0{ }^{\circ} \mathrm{C}$. Benzyl bromide (0.505 mL, $4.22 \mathrm{mmol}, 2.0$ equiv) and TBAl $(0.039 \mathrm{~g}, 0.10 \mathrm{mmol}, 0.05$ equiv) were then added and the resulting solution was warmed to ambient temperature. After being stirred for $4 \mathrm{~h}$ at 
ambient temperature, the reaction mixture was poured into a saturated aqueous $\mathrm{NH}_{4} \mathrm{Cl}$ solution $(50 \mathrm{~mL})$ and the resulting mixture was extracted with ether $(3 \times 50 \mathrm{~mL})$. The combined organic layers were washed with brine $(50 \mathrm{~mL})$, dried over $\mathrm{MgSO}_{4}$, filtered and concentrated in vacuo. Flash chromatography on silica gel, using ethyl acetate-hexanes $(1: 7 \rightarrow 1: 4)$ as eluant, provided benzyl ether (+)-S3 (1.29 g, $1.83 \mathrm{mmol}, 97 \%$ yield) as a colorless oil: $R_{f} 0.70$ (hexanes/ethyl acetate 4/1); $[\alpha]^{20}{ }_{D}+4.2\left(c\right.$ 1.0, $\left.\mathrm{CHCl}_{3}\right)$; IR (film) $3034(\mathrm{~m}), 2930$ (s), 2856 (s), $1954(w), 1659(w)$. 1589 (w), 1496 (m), 1454 (s), 1428 (s), 1111 (s), 984 (s), 736 (s) cm ${ }^{-1} ;{ }^{1} \mathrm{H}$ NMR (500 MHz, CDCl ${ }_{3}$ ) $\delta$ 7.75-7.74 (m, 2H), 7.69-7.67 (m, 2H), 7.41-7.25 (m, 11H), 7.13-7.11 (m, 2H), 7.06-7.02 (m, 3H), 5.92 (ddd, $J=10.0,4.9$ and $2.8 \mathrm{~Hz}, 1 \mathrm{H}), 5.62(\operatorname{app} \mathrm{dt}, J=10.1$ and $1.8 \mathrm{~Hz}, 1 \mathrm{H}), 4.64(\mathrm{~d}, J=11.4$ $\mathrm{Hz}, 1 \mathrm{H}), 4.54\left(\mathrm{~d}, J_{\mathrm{AB}}=12.1 \mathrm{~Hz}, 1 \mathrm{H}\right), 4.51\left(\mathrm{~d}, J_{\mathrm{AB}}=12.1 \mathrm{~Hz}, 1 \mathrm{H}\right), 4.35(\mathrm{~d}, J=11.4 \mathrm{~Hz}, 1 \mathrm{H}), 4.18-$ $4.13(\mathrm{~m}, 1 \mathrm{H}), 3.93-3.89(\mathrm{~m}, 1 \mathrm{H}), 3.84(\mathrm{dd}, J=11.0$ and $2.8 \mathrm{~Hz}, 1 \mathrm{H}), 3.65-3.59(\mathrm{~m}, 3 \mathrm{H}), 3.53(\mathrm{dd}$, $J=10.2$ and $5.3 \mathrm{~Hz}, 1 \mathrm{H}), 3.50-3.47(\mathrm{~m}, 1 \mathrm{H}), 3.39(\mathrm{~s}, 3 \mathrm{H}), 2.08-2.05(\mathrm{~m}, 1 \mathrm{H}), 1.98-1.95(\mathrm{~m}, 2 \mathrm{H})$, 1.85-1.64 (m, 5H), $1.02(\mathrm{~s}, 9 \mathrm{H}) ;{ }^{13} \mathrm{C}$ NMR $\left(125 \mathrm{MHz}, \mathrm{CDCl}_{3}\right) \delta 138.7,138.4,136.0,135.7,134.3$, $133.9,130.1,129.32,129.29,128.3,128.1,127.6,127.5,127.4,127.32,127.30,127.20,127.18$, $93.9,75.6,74.0,73.8,73.3,73.1,72.0,64.3,63.3,56.7,38.8,34.3,31.2,26.8,24.0,19.4,15.3$; high resolution mass spectrum (ESI) $\mathrm{m} / \mathrm{z} 729.3616\left[(\mathrm{M}+\mathrm{Na})^{+}\right.$; calcd for $\mathrm{C}_{44} \mathrm{H}_{54} \mathrm{O}_{8} \mathrm{NaSi}$ : 729.3587].

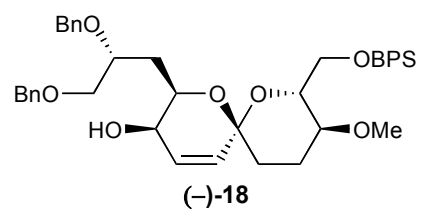

Alcohol (-)-18. To a solution of alkene (+)-S3 $(0.170 \mathrm{~g}, 0.240 \mathrm{mmol})$ in dioxane $(10 \mathrm{~mL})$ were added pyridine $\left(0.30 \mathrm{~mL}, 3.7 \mathrm{mmol}, 15\right.$ equiv) and $\mathrm{SeO}_{2}(0.20 \mathrm{~g}, 1.8 \mathrm{mmol}, 7.5$ equiv). With a microwave reactor, the mixture was stirred for $105 \mathrm{~min}$ at $120^{\circ} \mathrm{C}$. The resulting dark brown mixture was then poured into a saturated $\mathrm{NaHCO}_{3}$ aqueous solution $(20 \mathrm{~mL})$ and the reaction vial was rinsed with $\mathrm{Et}_{2} \mathrm{O}(10 \mathrm{~mL})$. After 20 min stirring at ambient temperature, the mixture was extracted with $\mathrm{Et}_{2} \mathrm{O}(30 \mathrm{~mL} \times 3)$. The combined organic layers were washed with brine $(20 \mathrm{~mL})$, 
dried over $\mathrm{MgSO}_{4}$, filtered and concentrated in vacuo. Flash chromatography on silica gel, using ethyl acetate-hexanes $(1: 4 \rightarrow 1: 1)$ as eluant, provided (-)-18 $(0.078 \mathrm{~g}, 0.108 \mathrm{mmol}, 45 \%$ yield $)$ as a pale yellow oil, (+)-S4 $(0.016 \mathrm{~g}, 0.022 \mathrm{mmol}, 9.2 \%$ yield) as a pale yellow oil, and the recovered starting material (16 mg, $0.023 \mathrm{mmol}, 9.4 \%$ ). For (-)-18: $R_{\mathrm{f}} 0.30$ (hexanes/ethyl acetate 2/1); $[\alpha]^{20}{ }_{D}-13.8\left(c\right.$ 1.0, $\mathrm{CHCl}_{3}$ ); IR (film) 3435 (br s), $3033(\mathrm{~m}), 2930$ (s), 2856 (s), 1960 (w), 1454 (s), 1428 (s), 1111 (s), 984 (s), 738 (s) cm ${ }^{-1} ;{ }^{1} \mathrm{H}$ NMR (500 MHz, $\left.\mathrm{CDCl}_{3}\right) \delta 7.72$ (dd, $J=7.9$ and 1.3 $\mathrm{Hz}, 2 \mathrm{H}), 7.68(\mathrm{dd}, \mathrm{J}=8.0$ and $1.4 \mathrm{~Hz}, 2 \mathrm{H}), 7.42-7.26(\mathrm{~m}, 11 \mathrm{H}), 7.17-7.12(\mathrm{~m}, 5 \mathrm{H}), 6.09(\mathrm{dd}, J=$ 9.9 and $5.5 \mathrm{~Hz}, 1 \mathrm{H}), 5.78$ (d, $J=10.0 \mathrm{~Hz}, 1 \mathrm{H}), 4.70(\mathrm{~d}, J=11.3 \mathrm{~Hz}, 1 \mathrm{H}), 4.53(\mathrm{~s}, 2 \mathrm{H}), 4.38(\mathrm{~d}, J$ $=11.4 \mathrm{~Hz}, 1 \mathrm{H}$ ), $4.15(\mathrm{ddd}, J=7.0,7.0$ and $2.1 \mathrm{~Hz}, 1 \mathrm{H}$ ), $3.84(\mathrm{dd}, J=11.0$ and $3.6 \mathrm{~Hz}, 1 \mathrm{H}$ ), 3.85$3.80(\mathrm{~m}, 1 \mathrm{H}), 3.70-3.67(\mathrm{~m}, 2 \mathrm{H}), 3.62-3.58(\mathrm{~m}, 3 \mathrm{H}), 3.44(\mathrm{ddd}, J=10.0,10.0$ and $4.2 \mathrm{~Hz}, 1 \mathrm{H})$, 3.39 (s, 3H), 2.13 (br d, J = 7.3 Hz, 1H), 2.10-2.06 (m, 1H), 1.97-1.89 (m, 3H), 1.85-1.80 (m, 1H), 1.67 (ddd, $J=13.4,13.4$ and $4.3 \mathrm{~Hz}, 1 \mathrm{H}), 1.03(\mathrm{~s}, 9 \mathrm{H}) ;{ }^{13} \mathrm{C}$ NMR $\left(125 \mathrm{MHz}, \mathrm{CDCl}_{3}\right) \delta 138.15$, $138.10,135.9,135.6,134.1,133.8,132.9,129.38,129.36,128.5,128.35,128.27,127.67$, $127.58,127.47,127.36,93.7,75.6,74.3,73.9,73.4,73.1,72.0,68.6,63.4,63.2,56.6,34.0,33.9$, 26.8, 23.8, 19.4; high resolution mass spectrum $(\mathrm{ESI}) \mathrm{m} / \mathrm{z} 745.3511\left[(\mathrm{M}+\mathrm{Na})^{+}\right.$; calcd for $\mathrm{C}_{44} \mathrm{H}_{54} \mathrm{O}_{7} \mathrm{NaSi}:$ 745.3537].

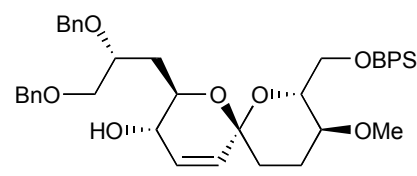

(+)-S4

For (+)-S4: $\mathrm{R}_{\mathrm{f}} 0.50$ (hexanes/ethyl acetate $\left.2 / 1\right) ;[\alpha]^{20}{ }_{\mathrm{D}}+20.2\left(\mathrm{c} \quad 0.6, \mathrm{CHCl}_{3}\right) ; \mathrm{IR}($ film) 3420 (br s), 3032 (m), 2930 (s), 2856 (s), 1723 (w), 1428 (s), 1362 (m), 1112 (s), 1018 (s), 738 (s) cm ${ }^{-1} ;{ }^{1} \mathrm{H}$ $\operatorname{NMR}\left(500 \mathrm{MHz}, \mathrm{CDCl}_{3}\right) \delta 7.74(\mathrm{dd}, J=8.0$ and $1.4 \mathrm{~Hz}, 2 \mathrm{H}), 7.70(\mathrm{dd}, \mathrm{J}=7.9$ and $1.4 \mathrm{~Hz}, 2 \mathrm{H}$ ), 7.42-7.26 (m, 11H), 7.18-7.10 (m, 5H), $5.88(\mathrm{dd}, J=10.1$ and $1.6 \mathrm{~Hz}, 1 \mathrm{H}), 5.60$ (dd, $J=10.1$ and $2.0 \mathrm{~Hz}, 1 \mathrm{H}), 4.66(\mathrm{~d}, J=11.4 \mathrm{~Hz}, 1 \mathrm{H}), 4.54\left(\mathrm{~d}, J_{\mathrm{AB}}=12.1 \mathrm{~Hz}, 1 \mathrm{H}\right), 4.51\left(\mathrm{~d}, J_{\mathrm{AB}}=12.1 \mathrm{~Hz}, 1 \mathrm{H}\right)$, $4.38(\mathrm{~d}, J=11.4 \mathrm{~Hz}, 1 \mathrm{H}), 3.91-3.85(\mathrm{~m}, 1 \mathrm{H}), 3.84-3.83(\mathrm{~m}, 2 \mathrm{H}), 3.77$ (ddd, $J=8.9,6.6$ and 4.9 $\mathrm{Hz}, 1 \mathrm{H}), 3.69(\mathrm{dd}, J=11.0$ and $1.4 \mathrm{~Hz}, 1 \mathrm{H}), 3.61-3.55(\mathrm{~m}, 3 \mathrm{H}), 3.46-3.40(\mathrm{~m}, 1 \mathrm{H}), 3.39(\mathrm{~s}, 3 \mathrm{H})$, 
$2.68(\mathrm{~d}, J=5.8 \mathrm{~Hz}, 1 \mathrm{H}), 2.12(\mathrm{ddd}, J=14.6,9.5$ and $4.8 \mathrm{~Hz}, 1 \mathrm{H}), 2.07-2.04(\mathrm{~m}, 1 \mathrm{H}), 1.83-1.74$ $(\mathrm{m}, 3 \mathrm{H}), 1.71-1.64(\mathrm{~m}, 1 \mathrm{H}), 1.04(\mathrm{~s}, 9 \mathrm{H}) ;{ }^{13} \mathrm{C} \mathrm{NMR}\left(125 \mathrm{MHz}, \mathrm{CDCl}_{3}\right) \delta 138.1,137.9,135.9$, $135.6,134.1,133.8,132.1,130.3,129.4,128.5,128.3,128.2,127.7,127.6,127.49,127.48$, $127.39,126.9,93.9,75.8,74.5,73.8,73.3,72.7,71.7,70.4,68.7,65.1,63.3,56.6,36.6,33.8$, 26.8, 23.5, 19.4; high resolution mass spectrum $(\mathrm{ESI}) \mathrm{m} / \mathrm{z} 745.3539\left[(\mathrm{M}+\mathrm{Na})^{+}\right.$; calcd for $\mathrm{C}_{44} \mathrm{H}_{54} \mathrm{O}_{7} \mathrm{NaSi}:$ 745.3537].

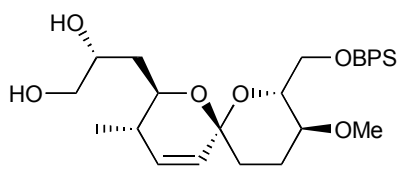

$(+)-19$

Diol (+)-19. To a solution of $\mathrm{PPh}_{3}(0.414 \mathrm{~g}, 1.58 \mathrm{mmol}, 3.0$ equiv) in benzene $(5 \mathrm{~mL})$ at ambient temperature was added DEAD $(0.25 \mathrm{~mL}, 1.58 \mathrm{mmol}, 3.0$ equiv). After $2 \mathrm{~min}$, a mixture of alcohol $(-)-18(0.380 \mathrm{~g}, 0.526 \mathrm{mmol})$ and bis (phenylsulfonyl)methane $(0.468 \mathrm{~g}, 1.58 \mathrm{mmol}, 3.0$ equiv) in benzene $(10 \mathrm{~mL})$ was added. The resulting reaction mixture was stirred for $1.5 \mathrm{~h}$ at ambient temperature and then the solvent was removed in vacuo. Flash chromatography on silica gel, using ethyl acetate-hexanes (1:2) as eluant, provided the desired product $(0.50 \mathrm{~g})$, contaminated with some DEAD. Without further purification, the mixture was used in the next step. It was dissolved in THF $(20 \mathrm{~mL})$ and $t-\mathrm{BuOH}(0.15 \mathrm{~mL}, 1.6 \mathrm{mmol}, 3.0$ equiv) was added. The resulting solution was cooled to $-78{ }^{\circ} \mathrm{C}$ and $\operatorname{LiDBB}(\sim 0.18 \mathrm{M}$ in THF, ca. $60 \mathrm{~mL})$ was added via cannula portionwise over 25 min until a dark green color persisted. After additional 5 min stirring at -78 ${ }^{\circ} \mathrm{C}$, a saturated $\mathrm{NH}_{4} \mathrm{Cl}$ aqueous solution $(30 \mathrm{~mL})$ and water $(30 \mathrm{~mL})$ were added. The resulting mixture was warmed to ambient temperature and extracted with ether $(3 \times 90 \mathrm{~mL})$. The combined organic layers were washed with brine $(50 \mathrm{~mL})$, dried over $\mathrm{MgSO}_{4}$, filtered and concentrated in vacuo. Flash chromatography on silica gel, using ethyl acetate-hexanes $(1: 10 \rightarrow$ ethyl acetate only) as eluant, provided diol $(+)-19(0.186 \mathrm{~g}, 0.344 \mathrm{mmol}, 65 \%$ yield over 2 steps) as a white foam: $R_{f} 0.30$ (hexanes/ethyl acetate $\left.1 / 1\right) ;[\alpha]_{D}^{20}+7.93\left(c \quad 0.74, \mathrm{CHCl}_{3}\right) ;$ IR (film) 3420 (br s), 3048 (w), 2931 (s), 2856 (s), 1653 (w), 1472 (m), $1428(\mathrm{~m}), 1155(\mathrm{~m}), 1017$ (s), 702 (s) $\mathrm{cm}^{-1} ;{ }^{1} \mathrm{H}$ NMR $\left(500 \mathrm{MHz}, \mathrm{CDCl}_{3}\right) \delta$ 7.78-7.75 (m, 4H), 7.42-7.34 (m, 6H), 5.75 (dd, $J=9.9$ and $1.8 \mathrm{~Hz}, 1 \mathrm{H}), 5.60(\mathrm{dd}, J=9.9$ and $2.5 \mathrm{~Hz}, 1 \mathrm{H}), 4.13-4.10(\mathrm{~m}, 1 \mathrm{H}), 3.91(\mathrm{dd}, J=10.7$ and $1.6 \mathrm{~Hz}$, 
1H), $3.87($ app td, $J=9.4$ and $2.5 \mathrm{~Hz}, 1 \mathrm{H}), 3.78(\mathrm{dd}, J=10.7$ and $5.7 \mathrm{~Hz}, 1 \mathrm{H}), 3.73$ (ddd, $J=9.4$, 5.7 and $1.6 \mathrm{~Hz}, 1 \mathrm{H}$ ), $3.65(\mathrm{dd}, J=11.0$ and $3.4 \mathrm{~Hz}, 1 \mathrm{H}$ ), $3.47(\mathrm{dd}, J=11.0$ and $7.0 \mathrm{~Hz}, 1 \mathrm{H}$ ), 3.33 (s, 3H), $3.20(\mathrm{app} t \mathrm{~d}, J=10.3$ and $3.9 \mathrm{~Hz}, 1 \mathrm{H}), 2.20-2.16(\mathrm{~m}, 1 \mathrm{H}), 2.10-2.04(\mathrm{~m}, 1 \mathrm{H}), 1.85-1.80$ (m, 2H), 1.75-1.65 (m, 2H), 1.55 (ddd, $J=11.4,9.0$ and $2.4 \mathrm{~Hz}, 1 \mathrm{H}), 1.04(\mathrm{~s}, 9 \mathrm{H}), 0.94$ (d, $J=7.2$ $\mathrm{Hz}, 3 \mathrm{H}) ;{ }^{13} \mathrm{C}$ NMR $\left(125 \mathrm{MHz}, \mathrm{CDCl}_{3}\right) \delta 135.85,135.83,134.4,133.8,133.7,129.44,129.41$, $128.65,127.5,93.5,74.6,74.4,70.7,68.4,67.1,64.1,56.4,35.5,33.8,33.7,26.8,24.0,19.3$, 16.7; high resolution mass spectrum (ESI) $\mathrm{m} / \mathrm{z} 563.2788\left[(\mathrm{M}+\mathrm{Na})^{+}\right.$; calcd for $\mathrm{C}_{31} \mathrm{H}_{44} \mathrm{O}_{6} \mathrm{NaSi}$ : 563.2805].

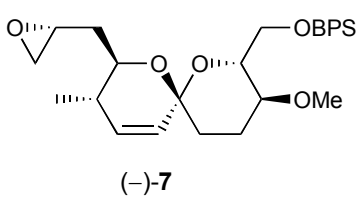

Epoxide (-)-7. To a solution of diol (+)-19 $(0.055 \mathrm{~g}, 0.10 \mathrm{mmol})$ in THF $(4 \mathrm{~mL})$ at $0{ }^{\circ} \mathrm{C}$ was added $\mathrm{NaH}(60 \%$ wt. in mineral oil, $0.012 \mathrm{~g}, 0.30 \mathrm{~mol}, 3.0$ equiv). The resulting mixture was then warmed to ambient temperature and stirred for $10 \mathrm{~min}$. The mixture was then cooled to $0{ }^{\circ} \mathrm{C}$ and $\mathrm{N}$-(2,4,6-triisopropylbenzenesulfonyl)imidazole $(0.0 .038 \mathrm{~g}, 0.11 \mathrm{mmol}, 1.1 \mathrm{equiv})$ was added in one portion. The reaction mixture was then allowed to warm to ambient temperature and stirred for $0.5 \mathrm{~h}$. The reaction was quenched with a saturated $\mathrm{NH}_{4} \mathrm{Cl}$ aqueous solution $(10 \mathrm{~mL})$ and extracted with $\mathrm{Et}_{2} \mathrm{O}(3 \times 10 \mathrm{~mL})$. The combined organic layers were washed with brine $(10 \mathrm{~mL})$, dried over $\mathrm{MgSO}_{4}$, filtered, and concentrated in vacuo. Flash chromatography on silica gel, using ethyl acetate-hexanes (1:5) as eluant, provided epoxide (-)-7 (0.051 g, $0.098 \mathrm{mmol}, 96 \%$ yield) as a colorless oil: $\mathrm{R}_{\mathrm{f}} 0.55$ (hexanes/ethyl acetate 3/1); $[\alpha]^{20}{ }_{\mathrm{D}}-2.9$ (c $0.14, \mathrm{CHCl}_{3}$ ); IR (film) 3047 (w), 2930 (s), 2856 (s), 1653 (w), 1472 (w), 1428 (m), 1112 (s), 1017 (s), 984 (s), 702 (s) cm ${ }^{-1} ;{ }^{1} \mathrm{H}$ NMR (500 MHz, $\left.\mathrm{CDCl}_{3}\right) \delta$ 7.77-7.74 (m, 4H), 7.41-7.32 (m, 6H), $5.72(\mathrm{dd}, J=9.9$ and $1.7 \mathrm{~Hz}, 1 \mathrm{H})$, $5.61(\mathrm{dd}, J=9.9$ and $2.5 \mathrm{~Hz}, 1 \mathrm{H}), 3.91-3.85(\mathrm{~m}, 2 \mathrm{H}), 3.76($ app td, $J=10.7$ and $2.4 \mathrm{~Hz}, 1 \mathrm{H}), 3.68$ (ddd, $J=9.6,4.0$ and $2.3 \mathrm{~Hz}, 1 \mathrm{H}$ ), 3.36 (s, 3H), 3.30 (app td, $J=10.3$ and $4.7 \mathrm{~Hz}, 1 \mathrm{H}$ ), 3.12-3.09 (m, 1H), $2.81($ app t, $J=4.6 \mathrm{~Hz}, 1 \mathrm{H}), 2.54(\mathrm{dd}, J=5.1$ and $2.6 \mathrm{~Hz}, 1 \mathrm{H}), 2.10-2.05(\mathrm{~m}, 2 \mathrm{H}), 1.94$ (ddd, $J=14.0,5.7$ and $2.4 \mathrm{~Hz}, 1 \mathrm{H}), 1.83($ app dt, $J=12.4$ and $3.3 \mathrm{~Hz}, 1 \mathrm{H}), 1.79-1.74(\mathrm{~m}, 1 \mathrm{H})$, 
1.69 (app qd, $J=13.3$ and $3.8 \mathrm{~Hz}, 1 \mathrm{H}), 1.55$ (ddd, $J=14.1,10.2$ and $6.0 \mathrm{~Hz}, 1 \mathrm{H}), 1.05(\mathrm{~s}, 9 \mathrm{H})$, $0.94(\mathrm{~d}, J=7.1 \mathrm{~Hz}, 3 \mathrm{H}) ;{ }^{13} \mathrm{C}$ NMR $\left(125 \mathrm{MHz}, \mathrm{CDCl}_{3}\right) \delta 135.9,135.8,134.2,133.92,133.88$, $129.3,129.0,127.45,127.38,93.3,74.4,74.3,70.9,63.8,56.6,49.9,47.8,36.5,34.4,33.9,26.8$, 24.1, 19.4, 16.7; high resolution mass spectrum $(\mathrm{ESI}) \mathrm{m} / \mathrm{z} 545.2723\left[(\mathrm{M}+\mathrm{Na})^{+}\right.$; calcd for $\mathrm{C}_{31} \mathrm{H}_{42} \mathrm{O}_{5} \mathrm{NaSi}:$ 545.2699].

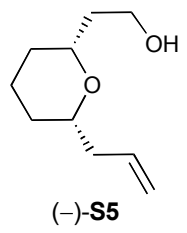

Alcohol (-)-S5. To a suspension of $\mathrm{LAH}\left(0.88 \mathrm{~g}, 23 \mathrm{~mol}, 2.6\right.$ equiv) in $\mathrm{Et}_{2} \mathrm{O}(100 \mathrm{~mL})$ at $0{ }^{\circ} \mathrm{C}$ was added a solution of ester (-)-20 (1.78 g, $9.98 \mathrm{mmol})$ in $\mathrm{Et}_{2} \mathrm{O}(10 \mathrm{~mL})$. The reaction mixture was stirred for $40 \mathrm{~min}$ at $0{ }^{\circ} \mathrm{C}$ and $\mathrm{Na}_{2} \mathrm{SO}_{4} \bullet 10 \mathrm{H}_{2} \mathrm{O}(7.4 \mathrm{~g}, 23 \mathrm{mmol}, 2.6$ equiv) was then added. The resulting mixture was stirred for $1 \mathrm{~h}$ at ambient temperature. The white suspended materials were filtered and repeatedly washed with $\mathrm{Et}_{2} \mathrm{O}$. The combined filtrates were concentrated in vacuo. Flash chromatography on silica gel, using ethyl acetate-hexanes (1:4) as eluant, provided alcohol (-)-S5 (1.46 g, $8.58 \mathrm{mmol}, 96 \%$ yield) as a colorless oil: $R_{\mathrm{f}} 0.30$ (hexanes/ethyl acetate 2/1); $[\alpha]_{D}^{20}-19.1$ (c 2.0, $\mathrm{CHCl}_{3}$ ); IR (film) 3407 (br s), $3076(\mathrm{~m}), 2936$ (s), 2858 (s), 1830 (w), 1642 (s), 1440 (s), 1331 (s), 1197 (s), 1084 (s), 914 (s) cm ${ }^{-1} ;{ }^{1} \mathrm{H}$ NMR (500 MHz, $\left.\mathrm{CDCl}_{3}\right) \delta 5.80$ (dddd, $J=17.0,10.2,6.9$ and $6.9 \mathrm{~Hz}, 1 \mathrm{H}), 5.10-5.05(\mathrm{~m}, 2 \mathrm{H}), 3.81-3.74(\mathrm{~m}, 2 \mathrm{H}), 3.57$ (dddd, $J=$ 11.3, 9.4, 2.4 and $2.4 \mathrm{~Hz}, 1 \mathrm{H}$ ), 3.41 (dddd, $J=11.2,7.4,5.4$ and $2.1 \mathrm{~Hz}, 1 \mathrm{H}), 3.12-3.10(\mathrm{~m}, 1 \mathrm{H})$, 2.27-2.18 (m, $2 \mathrm{H}), 1.86-1.81(\mathrm{~m}, 1 \mathrm{H}), 1.80-1.72(\mathrm{~m}, 1 \mathrm{H}), 1.67-1.48(\mathrm{~m}, 4 \mathrm{H}), 1.36-1.28(\mathrm{~m}, 1 \mathrm{H})$, 1.28-1.19 (m, 1H); ${ }^{13} \mathrm{C}$ NMR $\left(125 \mathrm{MHz}, \mathrm{CDCl}_{3}\right) \delta 134.9,117.0,79.4,77.3,62.1,40.9,37.8,31.4$, 30.9, 23.2; high resolution mass spectrum $(\mathrm{Cl}) \mathrm{m} / \mathrm{z} 171.1382\left[(\mathrm{M}+\mathrm{H})^{+}\right.$; calcd for $\mathrm{C}_{10} \mathrm{H}_{19} \mathrm{O}_{2}$ : 171.1385]. 


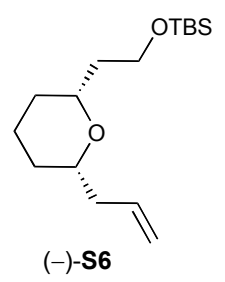

TBS ether (-)-S6. To a solution of $(+)-S 5(1.40 \mathrm{~g}, 8.22 \mathrm{mmol})$ in $\mathrm{CH}_{2} \mathrm{Cl}_{2}(15 \mathrm{~mL})$ at ambient temperature were added imidazole $(1.12 \mathrm{~g}, 16.4 \mathrm{mmol}, 2.0$ equiv), DMAP (0.10 g, $0.82 \mathrm{mmol}$, 0.10 equiv), and TBSCl (1.86 mL, $12.3 \mathrm{mmol}, 1.5$ equiv). After being stirred for $20 \mathrm{~min}$, the mixture was loaded on a silica gel column without work-up. Flash chromatography on silica gel, using ethyl acetate-hexanes (1:20) as eluant, provided (-)-S6 (2.27 g, $7.98 \mathrm{mmol}, 97 \%$ yield) as a colorless oil: $\mathrm{R}_{\mathrm{f}} 0.65$ (hexanes/ethyl acetate $7 / 1$ ); $[\alpha]^{20}{ }_{\mathrm{D}}-15\left(\mathrm{c} 1.0, \mathrm{CHCl}_{3}\right.$ ); IR (film) $3078(\mathrm{w})$, 2933 (s), 2857 (s), 1637 (w), 1469 (m), 1254 (m), 1089 (s), 835 (s), 776 (s) cm ${ }^{-1} ;{ }^{1}$ H NMR (500 $\mathrm{MHz}, \mathrm{CDCl}_{3}$ ) $\delta 5.84$ (dddd, $J=17.1,9.8,6.8$ and $\left.6.8 \mathrm{~Hz}, 1 \mathrm{H}\right), 5.05(\mathrm{br} \mathrm{d}, J=17.2 \mathrm{~Hz}, 1 \mathrm{H}$ ), 5.00 (br d, $J=10.1 \mathrm{~Hz}, 1 \mathrm{H}), 3.78-3.70(\mathrm{~m}, 1 \mathrm{H}), 3.69-3.66(\mathrm{~m}, 1 \mathrm{H}), 3.45-3.40(\mathrm{~m}, 1 \mathrm{H}), 3.32-3.27(\mathrm{~m}$, 1H), 2.29 (ddd, $J=13.9,6.6$ and $6.6 \mathrm{~Hz}, 1 \mathrm{H}), 2.15(\mathrm{ddd}, J=13.8,7.3$ and $6.3 \mathrm{~Hz}, 1 \mathrm{H}), 1.83-1.79$ $(\mathrm{m}, 1 \mathrm{H}), 1.70-1.44(\mathrm{~m}, 5 \mathrm{H}), 1.24-1.12(\mathrm{~m}, 2 \mathrm{H}), 0.90(\mathrm{~s}, 9 \mathrm{H}), 0.052(\mathrm{~s}, 3 \mathrm{H}), 0.047(\mathrm{~s}, 3 \mathrm{H}) ;{ }^{13} \mathrm{C} N M R$ $\left(125 \mathrm{MHz}, \mathrm{CDCl}_{3}\right) \delta 135.3,116.2,77.3,74.3,59.5,41.1,39.6,31.7,31.2,26.0,23.7,18.4,-5.3$, -5.4; high resolution mass spectrum $(\mathrm{ESI}) \mathrm{m} / \mathrm{z} 285.2256\left[(\mathrm{M}+\mathrm{H})^{+}\right.$; calcd for $\mathrm{C}_{16} \mathrm{H}_{33} \mathrm{O}_{2} \mathrm{Si}$ : 285.2253].

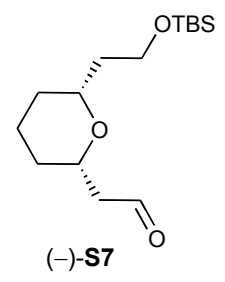

Aldehyde (-)-S7. To a solution of alkene (-)-S6 $(0.20 \mathrm{~g}, 0.70 \mathrm{mmol})$ in dioxane-water (4.5/1.5 $\mathrm{mL}$ ) at ambient temperature were added 2.6-lutidine (0.16 mmol, $1.4 \mathrm{mmol}, 2.0$ equiv), OsO4 (2.5 wt\% in 2-methyl-2-propanol, $0.18 \mathrm{~mL}, 0.014 \mathrm{mmol}, 0.02$ equiv) and $\mathrm{NalO}_{4}(0.60 \mathrm{~g}, 2.8 \mathrm{mmol}, 4.0$ equiv). After $2 \mathrm{~h}$ stirring at ambient temperature, water $(15 \mathrm{~mL})$ and $\mathrm{CH}_{2} \mathrm{Cl}_{2}(20 \mathrm{~mL})$ were added. The organic layer was separated and the aqueous layer was extracted with $\mathrm{CH}_{2} \mathrm{Cl}_{2}(10 \mathrm{~mL} X 2)$. 
The combined organic layers were washed with brine $(10 \mathrm{~mL})$, dried over $\mathrm{MgSO}_{4}$, filtered and concentrated in vacuo. Flash chromatography on silica gel, using ethyl acetate-hexanes $(1: 20 \rightarrow 1: 10)$ as eluant, provided aldehyde (-)-S7 $(0.12 \mathrm{~g}, 0.42 \mathrm{mmol}, 60 \%$ yield $)$ as a colorless oil: $R_{\mathrm{f}} 0.65$ (hexanes/ethyl acetate 4/1); $[\alpha]^{20}-25$ (c 0.68, $\mathrm{CHCl}_{3}$ ); IR (film) 2931 (s), 2857 (s), 2726 (w), 1729 (s), 1472 (m), 1256 (m), 1199 (w), 1096 (s), 836 (s), 776 (s) cm ${ }^{-1} ;{ }^{1}$ H NMR (500 $\left.\mathrm{MHz}, \mathrm{CDCl}_{3}\right) \delta 9.55(\mathrm{dd}, J=2.8$ and $1.8 \mathrm{~Hz}, 1 \mathrm{H}), 3.74(\mathrm{ddd}, J=10.0,8.3$ and $5.5 \mathrm{~Hz}, 1 \mathrm{H}$ ), 3.63 (ddd, $J=10.0,6.1$ and $5.0 \mathrm{~Hz}, 1 \mathrm{H}$ ), 3.46 (dddd, $J=12.8,7.7,4.6$ and $2.1 \mathrm{~Hz}, 1 \mathrm{H}$ ), 3.38 (dddd, $J$ $=10.8,8.4,3.9$ and 2.1, $1 \mathrm{H}$ ), $2.23(\mathrm{ddd}, J=15.9,8.0$ and $3.1 \mathrm{~Hz}, 1 \mathrm{H}$ ), 1.93 (ddd, $J=15.9,4.6$ and $1.8 \mathrm{~Hz}, 1 \mathrm{H}), 1.70-1.64(\mathrm{~m}, 1 \mathrm{H}), 1.63-1.56(\mathrm{~m}, 1 \mathrm{H}), 1.51-1.47(\mathrm{~m}, 1 \mathrm{H}), 1.29-1.25(\mathrm{~m}, 1 \mathrm{H})$, 1.24-1.17 (m, 1H), 1.16-1.11 (m, 1H), 1.06-1.00 (m, 1H), $0.99(\mathrm{~s}, 9 \mathrm{H}), 0.99-0.86(\mathrm{~m}, 1 \mathrm{H}), 0.08(\mathrm{~s}$, 3H), 0.07 (s, 3H); ${ }^{13} \mathrm{C}$ NMR (125 MHz, $\left.\mathrm{CDCl}_{3}\right) \delta 199.7,74.5,73.0,59.6,50.2,40.0,31.6,31.5$, 26.1, 23.7, 18.5, -5.2, -5.3; high resolution mass spectrum $(\mathrm{ESI}) \mathrm{m} / \mathrm{z} 287.2038\left[(\mathrm{M}+\mathrm{H})^{+}\right.$; calcd for $\left.\mathrm{C}_{15} \mathrm{H}_{31} \mathrm{O}_{3} \mathrm{Si}: 287.2042\right]$.

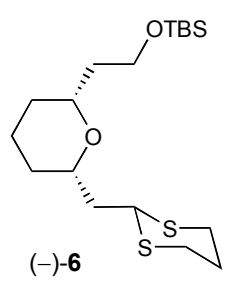

Dithiane (-)-6. To a solution of (-)-S7 $(0.480 \mathrm{~g}, 1.68 \mathrm{mmol})$ in $\mathrm{Et}_{2} \mathrm{O}(5 \mathrm{~mL})$ at $0{ }^{\circ} \mathrm{C}$ was added $1,3-$ propanedithiol $\left(0.30 \mathrm{~mL}, 2.98 \mathrm{mmol}, 1.8\right.$ equiv) followed by $\mathrm{MgBr}_{2}(0.464 \mathrm{~g}, 2.52 \mathrm{mmol}, 1.5$ equiv). The mixture was warmed to ambient temperature and stirred for $10 \mathrm{~min}$. A saturated $\mathrm{NaHCO}_{3}$ solution $(10 \mathrm{~mL})$ was then added and the mixture was extracted with $\mathrm{Et}_{2} \mathrm{O}(15 \mathrm{~mL} \times 2)$. The combined organic layers were washed with brine $(10 \mathrm{~mL})$, dried over $\mathrm{MgSO}_{4}$, filtered and concentrated in vacuo. Flash chromatography on silica gel, using ethyl acetate-hexanes (1:30) as eluant, provided dithiane (-)-6 $\left(0.450 \mathrm{~g}, 1.19 \mathrm{mmol}, 71 \%\right.$ yield) as a pale yellow oil: $R_{f} 0.55$ (hexanes/ethyl acetate 7/1); $[\alpha]^{20}{ }_{D}-15\left(c\right.$ 1.00, $\mathrm{CHCl}_{3}$ ); IR (film) 2932 (s), 2856 (s), 1468 (m), 1389 (m), 1253 (m), 1089 (s), 1010 (m), 955 (m), 836 (s), 776 (s) cm ${ }^{-1} ;{ }^{1} \mathrm{H}$ NMR (500 MHz, $\left.\mathrm{CDCl}_{3}\right) \delta 4.22(\mathrm{dd}, J=9.5$ and $4.9 \mathrm{~Hz}, 1 \mathrm{H}), 3.77-3.69(\mathrm{~m}, 2 \mathrm{H}), 3.56$ (dddd, $J=10.7,8.9,3.8$ and $1.6 \mathrm{~Hz}, 1 \mathrm{H}), 3.44-3.39(\mathrm{~m}, 1 \mathrm{H}), 2.91-2.80(\mathrm{~m}, 4 \mathrm{H}), 2.13-2.09(\mathrm{~m}, 1 \mathrm{H}), 1.95-1.86(\mathrm{~m}, 1 \mathrm{H}), 1.84-$ 
$1.75(\mathrm{~m}, 2 \mathrm{H}), 1.73-1.60(\mathrm{~m}, 2 \mathrm{H}), 1.56-1.48(\mathrm{~m}, 4 \mathrm{H}), 1.23-1.15(\mathrm{~m}, 2 \mathrm{H}), 0.90(\mathrm{~s}, 9 \mathrm{H}), 0.07(\mathrm{~s}, 3 \mathrm{H})$, 0.06 (s, 3H); ${ }^{13} \mathrm{C}$ NMR $\left(125 \mathrm{MHz}, \mathrm{CDCl}_{3}\right) \delta 74.5,73.5,59.8,43.6,42.3,39.7,31.7,31.6,30.4$, $30.1,26.1,26.0,23.6,18.4,-5.2$; high resolution mass spectrum $(\mathrm{Cl}) \mathrm{m} / \mathrm{z} 377.1982\left[(\mathrm{M}+\mathrm{H})^{+}\right.$; calcd for $\left.\mathrm{C}_{18} \mathrm{H}_{37} \mathrm{O}_{2} \mathrm{~S}_{2} \mathrm{Si}: 377.1971\right]$.

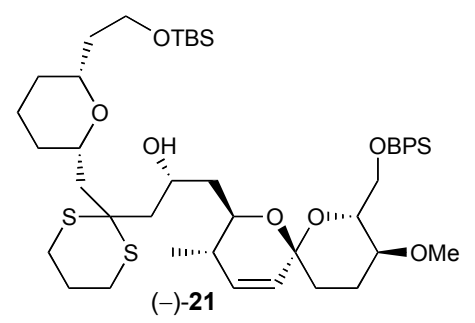

Alcohol (-)-21. To a solution of dithiane (-)-6 $(0.130 \mathrm{~g}, 0.345 \mathrm{mmol}, 2.3$ equiv) in THF $(2 \mathrm{~mL})$ at $78{ }^{\circ} \mathrm{C}$ was added $\mathrm{KO}-t$-Bu (1.0 M in THF, $0.35 \mathrm{~mL}, 0.35 \mathrm{mmol}, 2.3$ equiv) and then $n$-BuLi (2.2 M in hexane, $0.16 \mathrm{~mL}, 0.35 \mathrm{mmol}, 2.3$ equiv). The resulting solution was stirred for $30 \mathrm{~min}$ at $-78{ }^{\circ} \mathrm{C}$ and a solution of epoxide $(-)-7(0.078 \mathrm{~g}, 0.15 \mathrm{mmol})$ in THF $(1.5 \mathrm{~mL})$ was added. The resulting solution was slowly warmed to $0{ }^{\circ} \mathrm{C}$ over $1.5 \mathrm{~h}$ and stirred for an additional $30 \mathrm{~min}$ at $0{ }^{\circ} \mathrm{C}$ before a saturated aqueous $\mathrm{NH}_{4} \mathrm{Cl}$ solution $(5 \mathrm{~mL})$ was added. The resulting mixture was then extracted with $\mathrm{Et}_{2} \mathrm{O}(10 \mathrm{~mL} X 3)$. The combined organic layers were washed with brine $(10 \mathrm{~mL})$, dried over $\mathrm{MgSO}_{4}$, filtered and concentrated in vacuo. Flash chromatography on silica gel, using ethyl acetate-hexanes (1:5) as eluant, provided (-)-21 (0.116, $0.129 \mathrm{mmol}, 87 \%$ yield) as a white foam: $\mathrm{R}_{\mathrm{f}} 0.55$ (hexanes/ethyl acetate 4/1); $[\alpha]^{20}{ }_{\mathrm{D}}-12.1$ (c 2.17, $\mathrm{CHCl}_{3}$ ); IR (film) 3458 (br m), 3044 (w), 2930 (s), 2856 (s), 1655 (w), 1428 (m), 1255 (m), 1105 (s), $985(\mathrm{~m}), 836$ (s) cm ${ }^{-1} ;{ }^{1} \mathrm{H}$ NMR (500 $\left.\mathrm{MHz}, \mathrm{CDCl}_{3}\right) \delta$ 7.77-7.75 (m, 4H), 7.41-7.32 (m, 6H), $5.72(\mathrm{dd}, J=10.0$ and $1.5 \mathrm{~Hz}, 1 \mathrm{H}), 5.58(\mathrm{dd}$, $J=9.9$ and $2.4 \mathrm{~Hz}, 1 \mathrm{H}), 4.45(\operatorname{app~t}, J=9.1 \mathrm{~Hz}, 1 \mathrm{H}), 3.90-3.87(\mathrm{~m}, 2 \mathrm{H}), 3.83$ (ddd, $J=10.0,10.0$ and $1.8 \mathrm{~Hz}, 1 \mathrm{H}), 3.78-3.75(\mathrm{~m}, 1 \mathrm{H}), 3.72-3.65(\mathrm{~m}, 3 \mathrm{H}), 3.41-3.38(\mathrm{~m}, 1 \mathrm{H}), 3.36(\mathrm{~s}, 3 \mathrm{H}), 3.36-3.32$ (m, 1H), 2.93-2.74 (m, 4H), $2.29(\mathrm{dd}, J=15.0$ and $8.3 \mathrm{~Hz}, 1 \mathrm{H}), 2.22-2.17(\mathrm{~m}, 2 \mathrm{H}), 2.10(\mathrm{dd}, J=$ 14.9 and $1.5 \mathrm{~Hz}, 1 \mathrm{H}), 2.06-2.01(\mathrm{~m}, 2 \mathrm{H}), 1.99-1.78(\mathrm{~m}, 6 \mathrm{H}), 1.77-1.70(\mathrm{~m}, 1 \mathrm{H}), 1.69-1.62(\mathrm{~m}, 1 \mathrm{H})$, 1.59-1.50 (m, 5H), 1.31-1.23 (m, 1H), 1.22-1.12 (m, 1H), $1.04(\mathrm{~s}, 9 \mathrm{H}), 0.96(\mathrm{~d}, J=7.1 \mathrm{~Hz}, 3 \mathrm{H})$, $0.88(\mathrm{~s}, 9 \mathrm{H}), 0.041$ (s, 3H), $0.036(\mathrm{~s}, 3 \mathrm{H}) ;{ }^{13} \mathrm{C}$ NMR $\left(125 \mathrm{MHz}, \mathrm{CDCl}_{3}\right) \delta 136.0,135.8,134.3$, 
133.9, 129.3, 128.8, 127.4, 127.3, 93.4, 74.9, 74.5, 74.2, 74.0, 70.8, 65.1, 63.8, 60.3, 56.1, 51.9, $46.2,45.8,40.4,39.8,34.0,33.5,32.6,31.3,26.8,26.5,26.2,26.0,25.0,23.8(2 \mathrm{C}), 19.4,18.3$, 16.8, -5.2; high resolution mass spectrum $(\mathrm{ESI}) \mathrm{m} / \mathrm{z} 921.4631 \quad\left[(\mathrm{M}+\mathrm{Na})^{+}\right.$; calcd for $\mathrm{C}_{49} \mathrm{H}_{78} \mathrm{O}_{7} \mathrm{~S}_{2} \mathrm{Si}_{2} \mathrm{Na:}$ 921.4625].

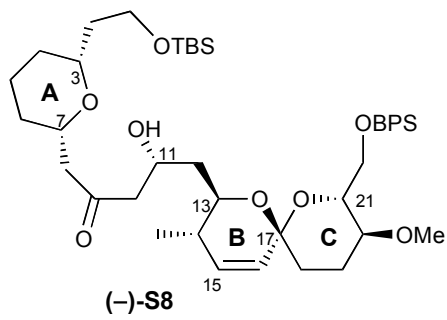

Ketone (-)-S8. To a solution of dithiane (-)-6 $(0.351 \mathrm{~g}, 0.390 \mathrm{mmol})$ in $\mathrm{THF} / \mathrm{H}_{2} \mathrm{O}(10 / 2 \mathrm{~mL})$ at $0{ }^{\circ} \mathrm{C}$ was added $\mathrm{CaCO}_{3}\left(0.156 \mathrm{~g}, 1.56 \mathrm{mmol}, 4.0\right.$ equiv) followed by $\mathrm{Hg}\left(\mathrm{ClO}_{4}\right)_{2} \bullet 3 \mathrm{H}_{2} \mathrm{O}(0.343 \mathrm{~g}, 0.859$ $\mathrm{mmol}, 2.2$ equiv). After being stirred for $5 \mathrm{~min}$ at $0{ }^{\circ} \mathrm{C}$, the reaction mixture was filtered through a short pad of Celite. The combined filtrates were concentrated in vacuo. Flash chromatography on silica gel, using ethyl acetate-hexanes (1:2) as eluant, provided ketone (-)-S8 (0.310 g, 0.383 mmol, $98 \%$ yield) as a pale yellow oil: $R_{f} 0.40$ (hexanes/ethyl acetate $3 / 1$ ); $[\alpha]^{20}{ }_{D}-9.1$ (c 0.033 , $\mathrm{CHCl}_{3}$ ); IR (film) 3523 (br m), 3048 (w), 2931 (s), 2856 (s), 1710 (m), 1472 (m), 1428 (m), 1255 (m), 1105 (s), $1016(\mathrm{~m}), 985(\mathrm{~m}), 836(\mathrm{~m}), 702(\mathrm{~m}) \mathrm{cm}^{-1} ;{ }^{1} \mathrm{H}$ NMR $\left(500 \mathrm{MHz}, \mathrm{CDCl}_{3}\right) \delta$ 7.77-7.75 (m, 4H), 7.41-7.32 (m, 6H), $5.73(\mathrm{dd}, J=10.0$ and $1.7 \mathrm{~Hz}, 1 \mathrm{H}), 5.58(\mathrm{dd}, J=10.0$ and $2.5 \mathrm{~Hz}$, 1H), 4.43-4.37 (m, 1H), 3.89-3.82 (m, 3H), 3.78-3.73 (m, 1H), 3.69-3.64 (m, 3H), 3.46-3.41 (m, 1H), 3.35 (s, 3H), 3.29 (ddd, $J=10.2,10.2$ and $4.3 \mathrm{~Hz}, 1 \mathrm{H}), 2.71-2.61(\mathrm{~m}, 3 \mathrm{H}), 2.45$ (dd, $J=15.4$ and $5.3 \mathrm{~Hz}, 1 \mathrm{H}), 2.21-2.16(\mathrm{~m}, 1 \mathrm{H}), 2.08-2.04(\mathrm{~m}, 1 \mathrm{H}), 1.84-1.80(\mathrm{~m}, 3 \mathrm{H}), 1.79-1.49(\mathrm{~m}, 8 \mathrm{H})$, 1.22-1.15 (m, 2H), $1.04(\mathrm{~s}, 9 \mathrm{H}), 0.96(\mathrm{~d}, J=7.1 \mathrm{~Hz}, 3 \mathrm{H}), 0.88(\mathrm{~s}, 9 \mathrm{H}), 0.041$ (s, 3H), $0.036(\mathrm{~s}$, $3 \mathrm{H}) ;{ }^{13} \mathrm{C}$ NMR $\left(125 \mathrm{MHz}, \mathrm{CDCl}_{3}\right) \delta 209.7,135.9,135.8,134.2,134.1,133.9,129.3,128.7,127.5$, $127.4,93.5,74.8,74.28,74.27,74.0,71.1,64.7,63.8,59.6,56.4,51.0,50.3,39.6,39.1,33.9$, $33.6,31.5,31.4,26.8,26.0,24.0,23.5,19.4,18.3,16.8,-5.3$; high resolution mass spectrum (ESI) $\mathrm{m} / \mathrm{z} 831.4677$ [(M+Na) ${ }^{+}$; calcd for $\mathrm{C}_{46} \mathrm{H}_{72} \mathrm{O}_{8} \mathrm{Si}_{2} \mathrm{Na}$ : 831.4663]. 


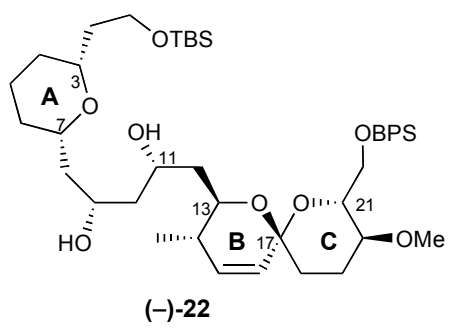

Anti diol (-)-22. To a solution of $\mathrm{Me}_{4} \mathrm{NHB}(\mathrm{OAc})_{3}\left(1.0 \mathrm{~g}, 3.8 \mathrm{mmol}, 10\right.$ equiv) in $\mathrm{CH}_{3} \mathrm{CN}(6 \mathrm{~mL})$ at ambient temperature was added $\mathrm{AcOH}(3 \mathrm{~mL})$. After being stirred for 30 min at ambient temperature, the mixture was cooled to $-35{ }^{\circ} \mathrm{C}$ and a solution of ketone (-)-S8 $(0.310 \mathrm{~g}, 0.383$ $\mathrm{mmol})$ in $\mathrm{CH}_{3} \mathrm{CN}(3 \mathrm{~mL})$ was added slowly. The resulting reaction mixture was stirred for $36 \mathrm{~h}$ at $35{ }^{\circ} \mathrm{C}$ and $0.5 \mathrm{~N}$ aqueous sodium potassium tartrate solution ( $8 \mathrm{~mL}, 4 \mathrm{mmol}$ ) was then added. The resulting mixture was allowed to warm to ambient temperature and extracted with $\mathrm{CH}_{2} \mathrm{Cl}_{2}(30$ $\mathrm{mL} X$ 3). The combined organic layers were washed with a saturated $\mathrm{NaHCO}_{3}$ solution $(30 \mathrm{~mL})$ and brine $(30 \mathrm{~mL})$, dried over $\mathrm{MgSO}_{4}$, filtered and concentrated in vacuo. Flash chromatography on silica gel, using ethyl acetate-hexanes (1:2) as eluant, provided syn diol (-)-S9 (0.065 g, 0.080 $\mathrm{mmol}, 21 \%$ yield $)$ as a colorless oil followed by anti diol (-)-22 (0.220 g, $0.271 \mathrm{mmol}, 71 \%$ yield) as a pale yellow oil. For anti diol (-)-22: $R_{f} 0.30$ (hexanes/ethyl acetate $\left.3 / 1\right) ;[\alpha]^{20}-9.7(c 0.70$, $\mathrm{CHCl}_{3}$ ); IR (film) 3446 (br s), 3048 (w), 2930 (s), 2856 (s), 1653 (w), 1472 (m), 1428 (m), 1256 (m), 1111 (s), $1016(\mathrm{~m}), 984(\mathrm{~m}), 836(\mathrm{~m}), 702(\mathrm{~m}) \mathrm{cm}^{-1} ;{ }^{1} \mathrm{H}$ NMR $\left(500 \mathrm{MHz}, \mathrm{CDCl}_{3}\right) \delta$ 7.78-7.74 $(\mathrm{m}, 4 \mathrm{H}), 7.41-7.33(\mathrm{~m}, 6 \mathrm{H}), 5.74(\mathrm{dd}, J=9.9$ and $1.6 \mathrm{~Hz}, 1 \mathrm{H}), 5.59(\mathrm{dd}, J=9.9$ and $2.4 \mathrm{~Hz}, 1 \mathrm{H})$, 4.35-4.30 (m, 1H), 4.26-4.21 (m, 1H), $3.88(\mathrm{dd}, J=10.8$ and $1.5 \mathrm{~Hz}, 1 \mathrm{H}), 3.88-3.84(\mathrm{~m}, 1 \mathrm{H}), 3.80$ (dd, $J=10.9$ and $5.3 \mathrm{~Hz}, 1 \mathrm{H}), 3.75-3.71(\mathrm{~m}, 2 \mathrm{H}), 3.70-3.61(\mathrm{~m}, 3 \mathrm{H}), 3.48-3.44(\mathrm{~m}, 1 \mathrm{H}), 3.42(\mathrm{br} \mathrm{s}$, 1H), $3.32(\mathrm{~s}, 3 \mathrm{H}), 3.24(\mathrm{ddd}, J=9.9,9.9$ and $4.3 \mathrm{~Hz}, 1 \mathrm{H}), 2.27-2.23(\mathrm{~m}, 1 \mathrm{H}), 2.07-2.04(\mathrm{~m}, 1 \mathrm{H})$, 1.89 (ddd, $J=12.8,9.8$ and $2.9 \mathrm{~Hz}, 1 \mathrm{H}), 1.84-1.81(\mathrm{~m}, 2 \mathrm{H}), 1.75-1.48(\mathrm{~m}, 12 \mathrm{H}), 1.44-1.36(\mathrm{~m}$, 1H), 1.25-1.20 (m, 1H), $1.04(\mathrm{~s}, 9 \mathrm{H}), 0.94(\mathrm{~d}, J=7.1 \mathrm{~Hz}, 3 \mathrm{H}), 0.88(\mathrm{~s}, 9 \mathrm{H}), 0.043(\mathrm{~s}, 3 \mathrm{H}), 0.038$ (s, 3H); ${ }^{13} \mathrm{C}$ NMR $\left(125 \mathrm{MHz}, \mathrm{CDCl}_{3}\right) \delta 135.9,135.8,134.4,134.0,133.8,129.38,129.36,128.6$, $127.44,127.43,93.5,75.7,74.7,74.4,74.2,71.5,66.0,65.7,63.9,59.5,56.3,43.9,42.6,39.6$, $39.2,33.8,33.3,31.6,31.1,26.8,25.9,24.0,23.6,19.3,18.3,16.8,-5.3$; high resolution mass spectrum (ESI) m/z 833.4786 [(M+Na) $)^{+}$; calcd for $\mathrm{C}_{46} \mathrm{H}_{74} \mathrm{O}_{8} \mathrm{Si}_{2} \mathrm{Na:}$ 833.4820]. 


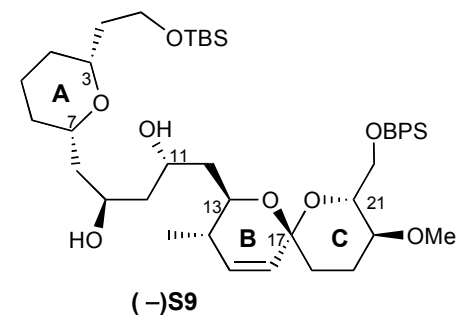

For syn diol (-)-S9: $\mathrm{R}_{\mathrm{f}} 0.45$ (hexanes/ethyl acetate $3 / 1$ ); $[\alpha]^{20}{ }_{\mathrm{D}}-4.8\left(\mathrm{c} 1.0, \mathrm{CHCl}_{3}\right.$ ); IR (film) 3465 (br s), 3047 (w), 2933 (s), 2857 (s), 1652 (w), $1472(\mathrm{~m}), 1430(\mathrm{~m}), 1254$ (m), 1107 (s), $1016(\mathrm{~m})$, $984(\mathrm{~m}), 836(\mathrm{~m}), 704(\mathrm{~m}) \mathrm{cm}^{-1} ;{ }^{1} \mathrm{H}$ NMR $\left(500 \mathrm{MHz}, \mathrm{CDCl}_{3}\right) \delta$ 7.77-7.75 (m, 4H), 7.41-7.33 (m, $6 \mathrm{H}), 5.71(\mathrm{dd}, J=9.9$ and $1.6 \mathrm{~Hz}, 1 \mathrm{H}), 5.58(\mathrm{dd}, J=9.9$ and $2.4 \mathrm{~Hz}, 1 \mathrm{H}), 4.19-4.13(\mathrm{~m}, 1 \mathrm{H}), 4.12-$ $4.07(\mathrm{~m}, 1 \mathrm{H}), 3.90-3.85(\mathrm{~m}, 2 \mathrm{H}), 3.80(\mathrm{ddd}, J=9.6,9.6$ and $2.0 \mathrm{~Hz}, 1 \mathrm{H}), 3.71-3.65(\mathrm{~m}, 3 \mathrm{H}), 3.60-$ $3.55(\mathrm{~m}, 1 \mathrm{H}), 3.55-3.49(\mathrm{~m}, 1 \mathrm{H}), 3.36(\mathrm{~s}, 3 \mathrm{H}), 3.33(\mathrm{ddd}, J=10.3,10.3$ and $4.6 \mathrm{~Hz}, 1 \mathrm{H}), 2.13-$ $2.04(\mathrm{~m}, 2 \mathrm{H}), 1.83-1.80(\mathrm{~m}, 3 \mathrm{H}), 1.80-1.50(\mathrm{~m}, 11 \mathrm{H}), 1.26-1.20(\mathrm{~m}, 3 \mathrm{H}), 1.04(\mathrm{~s}, 9 \mathrm{H}), 0.95(\mathrm{~d}, J=$ $7.1 \mathrm{~Hz}, 3 \mathrm{H}), 0.88(\mathrm{~s}, 9 \mathrm{H}), 0.04(\mathrm{~s}, 3 \mathrm{H}), 0.03(\mathrm{~s}, 3 \mathrm{H}) ;{ }^{13} \mathrm{C} \mathrm{NMR}\left(125 \mathrm{MHz}, \mathrm{CDCl}_{3}\right) \delta$ 136.0, 135.8, $134.3,134.2,133.9,129.3,128.8,127.44,127.37,93.4,79.0,74.9,74.3,74.1,72.7,71.1,69.1$, $63.8,59.6,56.5,44.5,43.3,40.4,39.4,34.2,34.0,31.9,31.4,26.8,26.0,24.2,23.4,19.4,18.3$, 16.9, -5.4; high resolution mass spectrum (ESI) $\mathrm{m} / \mathrm{z} 833.4840\left[(\mathrm{M}+\mathrm{Na})^{+}\right.$; calcd for $\mathrm{C}_{46} \mathrm{H}_{74} \mathrm{O}_{8} \mathrm{Si}_{2} \mathrm{Na}$ : 833.4820].

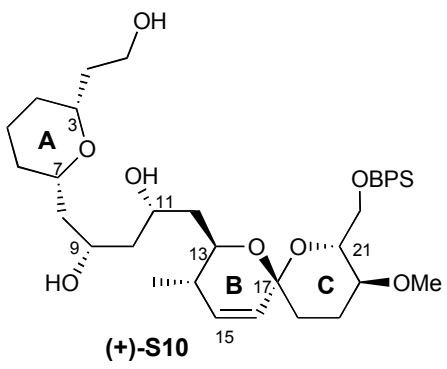

Triol (+)-S10. To a solution of (-)-22 $(0.040 \mathrm{~g}, 0.050 \mathrm{mmol})$ in $\mathrm{CH}_{2} \mathrm{Cl}_{2}-\mathrm{MeOH}(1 / 3 \mathrm{~mL})$ at $0{ }^{\circ} \mathrm{C}$ was added CSA (11 mg, $0.050 \mathrm{mmol}, 1.0$ equiv). The mixture was stirred for $30 \mathrm{~min}$ at $0{ }^{\circ} \mathrm{C}$ and the reaction was quenched with triethylamine $(0.05 \mathrm{~mL})$. The resulting mixture was concentrated in vacuo. Flash chromatography on silica gel, using ethyl acetate-hexanes $(1: 3 \rightarrow 1: 1)$ as eluant, provided triol (+)-S10 (34 mg, $49 \mu \mathrm{mol}, 98 \%$ yield) as a white foam oil: $R_{f} 0.20$ (hexanes/acetone 
3/1); $[\alpha]^{20}{ }_{D}+2.12\left(c 0.708, \mathrm{CHCl}_{3}\right) ; \mathrm{IR}$ (film) 3419 (br s), 3071 (w), $3048(w), 2932$ (s), 2856 (s), 1659 (w), 1428 (s), $1382(\mathrm{~m}), 1196(\mathrm{~m}), 1105$ (s), $985(\mathrm{~m}), 917(\mathrm{~m}), 702(\mathrm{~s}) \mathrm{cm}^{-1} ;{ }^{1} \mathrm{H}$ NMR (500 $\left.\mathrm{MHz}, \mathrm{CDCl}_{3}\right) \delta$ 7.76-7.73 (m, 4H), 7.41-7.32 (m, 6H), $5.73(\mathrm{dd}, J=9.9$ and $1.7 \mathrm{~Hz}, 1 \mathrm{H}), 5.59(\mathrm{dd}$, $J=10.0$ and $2.5 \mathrm{~Hz}, 1 \mathrm{H}), 4.35(\operatorname{app~t}, J=9.0 \mathrm{~Hz}, 1 \mathrm{H}), 4.14-4.10(\mathrm{~m}, 1 \mathrm{H}), 3.88-3.80(\mathrm{~m}, 2 \mathrm{H}), 3.80-$ $3.66(\mathrm{~m}, 4 \mathrm{H}), 3.62-3.57(\mathrm{~m}, 1 \mathrm{H}), 3.56-3.50(\mathrm{~m}, 1 \mathrm{H}), 3.34(\mathrm{~s}, 3 \mathrm{H}), 3.34-3.29(\mathrm{~m}, 1 \mathrm{H}), 2.20-2.15(\mathrm{~m}$, 1H), 2.09-2.04 (m, 1H), 1.89 (ddd, $J=13.9,10.1$ and $2.7 \mathrm{~Hz}, 1 \mathrm{H}), 1.84-1.72(\mathrm{~m}, 3 \mathrm{H}), 1.69-1.41$ $(\mathrm{m}, 12 \mathrm{H}), 1.29-1.21(\mathrm{~m}, 2 \mathrm{H}), 1.04(\mathrm{~s}, 9 \mathrm{H}), 0.94(\mathrm{~d}, J=7.1 \mathrm{~Hz}, 3 \mathrm{H}) ;{ }^{13} \mathrm{C}$ NMR $\left(125 \mathrm{MHz}, \mathrm{CDCl}_{3}\right) \delta$ 135.9, 135.8, 134.4, 129.4, 128.5, 127.5, 127.4, 93.4, 76.5, 74.9, 74.3, 73.8, 70.8, 65.3, 64.9, $63.7,60.1,56.3,44.3,43.5,39.5,38.5,33.8,33.7,31.62,31.57,26.8,24.0,23.6,19.3,16.8$; high resolution mass spectrum (ESI) $\mathrm{m} / \mathrm{z} 719.3982$ [(M+Na) ${ }^{+}$; calcd for $\mathrm{C}_{40} \mathrm{H}_{60} \mathrm{O}_{8} \mathrm{SiNa}$ : 719.3955].

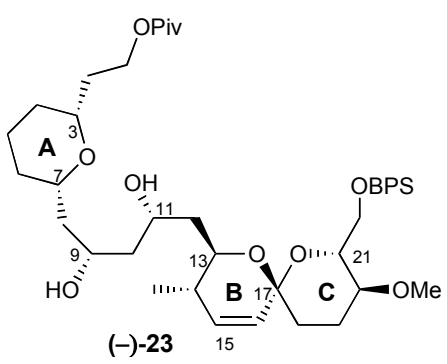

Pivaloate ester (-)-23. To a solution of triol (+)-S10 $(0.174 \mathrm{~g}, 0.250 \mathrm{mmol})$ in $\mathrm{CH}_{2} \mathrm{Cl}_{2}(8 \mathrm{~mL})$ at 0 ${ }^{\circ} \mathrm{C}$ was added DMAP (61 mg, $0.50 \mathrm{mmol}, 2.0$ equiv) followed by $\mathrm{PivCl}(34 \mu \mathrm{L}, 0.28 \mathrm{mmol}, 1.1$ equiv). The resulting solution was stirred for $10 \mathrm{~min}$ at $0{ }^{\circ} \mathrm{C}$ and a saturated aqueous $\mathrm{NH}_{4} \mathrm{Cl}$ solution $(5 \mathrm{~mL})$ was added. The organic layer was separated and the aqueous layer was extracted with $\mathrm{CH}_{2} \mathrm{Cl}_{2}(5 \mathrm{~mL} \times 3)$. The combined organic layers were washed with brine $(5 \mathrm{~mL})$, dried over $\mathrm{MgSO}_{4}$, filtered and concentrated in vacuo. Flash chromatography on silica gel, using ethyl acetate-hexanes (1:1) as eluant, provided diol (-)-23 (0.170 g, $0.218 \mathrm{mmol}, 87 \%$ yield) as a white solid: $\mathrm{R}_{\mathrm{f}} 0.45$ (hexanes/acetone 1/1); m.p. $98-99{ }^{\circ} \mathrm{C}$; $[\alpha]^{20}{ }_{\mathrm{D}}-5.4\left(\mathrm{c} 1.0, \mathrm{CHCl}_{3}\right)$; IR (film) 3436 (br s), 3047 (w), 2933 (s), 1727 (s), 1457 (m), 1428 (m), 1286 (m), 1156 (s), 1111 (s), 984 (m), $917(\mathrm{~m}), 702(\mathrm{~s}) \mathrm{cm}^{-1} ;{ }^{1} \mathrm{H}$ NMR $\left(500 \mathrm{MHz}, \mathrm{CDCl}_{3}\right) \delta$ 7.77-7.74 (m, 4H), 7.41-7.32 (m, 6H), $5.74(\mathrm{dd}, J=9.9$ and $1.8 \mathrm{~Hz}, 1 \mathrm{H}), 5.58(\mathrm{dd}, J=9.9$ and $2.5 \mathrm{~Hz}, 1 \mathrm{H}), 4.35-4.30(\mathrm{~m}, 1 \mathrm{H}), 4.30$ (ddd, $J=10.9,7.6$ and $6.5 \mathrm{~Hz}, 1 \mathrm{H}), 4.26-4.21(\mathrm{~m}, 1 \mathrm{H}), 4.06(\operatorname{app} \mathrm{dt}, J=11.2$ and $5.8 \mathrm{~Hz}, 1 \mathrm{H}), 3.88$ (dd, 
$J=10.8$ and $1.7 \mathrm{~Hz}, 1 \mathrm{H}), 3.89-3.84(\mathrm{~m}, 1 \mathrm{H}), 3.80(\mathrm{dd}, J=10.8$ and $5.3 \mathrm{~Hz}, 1 \mathrm{H}), 3.73(\mathrm{ddd}, J=$ 9.5, 5.2 and $1.7 \mathrm{~Hz}, 1 \mathrm{H}), 3.62-3.57(\mathrm{~m}, 1 \mathrm{H}), 3.55(\mathrm{br} \mathrm{s}, 1 \mathrm{H}), 3.42(\mathrm{br} \mathrm{s}, 1 \mathrm{H}), 3.38-3.33(\mathrm{~m}, 1 \mathrm{H})$, $3.32(\mathrm{~s}, 3 \mathrm{H}), 3.24$ (ddd, $J=9.9,9.9$ and $4.4 \mathrm{~Hz}, 1 \mathrm{H}), 2.28-2.23(\mathrm{~m}, 1 \mathrm{H}), 2.08-2.03(\mathrm{~m}, 1 \mathrm{H}), 1.90$ (ddd, $J=14.5,9.7$ and $2.9 \mathrm{~Hz}, 1 \mathrm{H}), 1.85-1.79(\mathrm{~m}, 2 \mathrm{H}), 1.77-1.47(\mathrm{~m}, 12 \mathrm{H}), 1.35$ (ddd, $\mathrm{J}=15.0$, 11.1 and $3.9 \mathrm{~Hz}, 1 \mathrm{H}), 1.23-1.18(\mathrm{~m}, 1 \mathrm{H}), 1.18(\mathrm{~s}, 9 \mathrm{H}), 1.03(\mathrm{~s}, 9 \mathrm{H}), 0.94(\mathrm{~d}, J=7.1 \mathrm{~Hz}, 3 \mathrm{H}) ;{ }^{13} \mathrm{C}$ NMR $\left(125 \mathrm{MHz}, \mathrm{CDCl}_{3}\right) \delta 178.6,135.9,135.8,134.4,133.8,129.38,129.36,128.6,127.45$, $127.43,93.5,75.4,74.5,74.4,74.3,71.6,65.79,65.74,64.0,60.9,56.3,43.9,43.1,39.1,38.7$, $35.6,33.8,33.3,31.5,31.3,27.2,26.8,24.0,23.6,19.3$, 16.8; high resolution mass spectrum (ESI) $\mathrm{m} / \mathrm{z} 803.4540\left[(\mathrm{M}+\mathrm{Na})^{+}\right.$; calcd for $\left.\mathrm{C}_{45} \mathrm{H}_{68} \mathrm{O}{ }_{9} \mathrm{SiNa}: 803.4530\right]$.

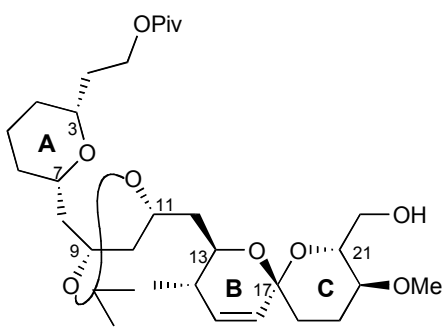

$(+)-S 11$

Acetonide (+)-S11. To a solution of diol (-)-23 (0.170 g, $0.218 \mathrm{mmol})$ in acetone/2,2dimethoxypropane $(5 / 1 \mathrm{~mL})$ at ambient temperature was added $\mathrm{pTsOH} \bullet \mathrm{H}_{2} \mathrm{O}(4.2 \mathrm{mg}, 0.022,0.10$ equiv). After being stirred for 5 min, the reaction mixture was poured into a saturated $\mathrm{NaHCO}_{3}$ solution $(20 \mathrm{~mL})$ and the resulting mixture was extracted with $\mathrm{Et}_{2} \mathrm{O}(30 \mathrm{~mL} \times 3)$. The combined organic layers were washed with brine $(20 \mathrm{~mL})$, dried over $\mathrm{MgSO}_{4}$, filtered and concentrated in vacuo. The resulting residue was dissolved in THF $(10 \mathrm{~mL})$ at ambient temperature and TBAF (1.0 M in THF, $2 \mathrm{~mL}, 2.0 \mathrm{mmol}, 9.2$ equiv) was added. After being stirred for 3 days at ambient temperature, the reaction mixture was poured into a saturated $\mathrm{NH}_{4} \mathrm{Cl}$ aqueous solution $(20 \mathrm{~mL})$ and the resulting mixture was extracted with $\mathrm{Et}_{2} \mathrm{O}(30 \mathrm{~mL} \times 3)$. The combined organic layers were washed with brine $(20 \mathrm{~mL})$, dried over $\mathrm{MgSO}_{4}$, filtered and concentrated in vacuo. Flash chromatography on silica gel, using ethyl acetate-hexanes (1.5:1) as eluant, provided (+)-S11 ( $0.105 \mathrm{~g}, 0.180 \mathrm{mmol}, 83 \%$ yield over 2 steps) as a colorless oil: $R_{f} 0.45$ (hexanes/ethyl acetate 1/1); $[\alpha]^{20}{ }_{D}+85.5\left(c\right.$ 1.0, $\left.\mathrm{C}_{6} \mathrm{H}_{6}\right) ;$ IR (film) 3501 (br s), 3032 (w), 2936 (s), 1729 (s), 1458 (m), 1379 
(m), 1285 (m), 1225 (s), 1160 (s), 1016 (s) cm ${ }^{-1} ;{ }^{1} \mathrm{H}$ NMR (500 MHz, $\left.\mathrm{CDCl}_{3}\right) \delta 5.69$ (dd, J = 10.2 and $1.2 \mathrm{~Hz}, 1 \mathrm{H}), 5.52(\mathrm{dd}, J=10.0$ and $2.5 \mathrm{~Hz}, 1 \mathrm{H}), 4.24-4.19(\mathrm{~m}, 1 \mathrm{H}), 4.15-4.06(\mathrm{~m}, 3 \mathrm{H}), 3.74-$ $3.67(\mathrm{~m}, 3 \mathrm{H}), 3.57(\operatorname{app~t}, J=9.6 \mathrm{~Hz}, 1 \mathrm{H}), 3.49-3.44(\mathrm{~m}, 1 \mathrm{H}), 3.40-3.36(\mathrm{~m}, 1 \mathrm{H}), 3.36(\mathrm{~s}, 3 \mathrm{H})$, $3.21(\mathrm{ddd}, J=9.7,9.7$ and $4.6 \mathrm{~Hz}, 1 \mathrm{H}), 2.06-2.02(\mathrm{~m}, 1 \mathrm{H}), 1.99-1.95(\mathrm{~m}, 1 \mathrm{H}), 1.88(\mathrm{dd}, J=14.3$ and $9.5 \mathrm{~Hz}, 1 \mathrm{H}), 1.80-1.60(\mathrm{~m}, 8 \mathrm{H}), 1.59-1.44(\mathrm{~m}, 8 \mathrm{H}), 1.32(\mathrm{~s}, 3 \mathrm{H}), 1.30(\mathrm{~s}, 3 \mathrm{H}), 1.17(\mathrm{~s}, 9 \mathrm{H})$, $0.97(\mathrm{~d}, J=7.2 \mathrm{~Hz}, 3 \mathrm{H}) ;{ }^{13} \mathrm{C}$ NMR $\left(125 \mathrm{MHz}, \mathrm{CDCl}_{3}\right) \delta 178.4,135.0,128.1,100.0,93.4,75.5$, $74.3,73.9,72.7,70.5,63.7,62.9,62.5,61.3,56.3,42.7,40.9,39.2,38.7,35.7,34.6,34.2,32.0$, $31.8,27.2,25.5,25.1,23.62,23.60,16.8$; high resolution mass spectrum (ESI) m/z 605.3665 $\left[(\mathrm{M}+\mathrm{Na})^{+}\right.$; calcd for $\left.\mathrm{C}_{32} \mathrm{H}_{54} \mathrm{O}_{9} \mathrm{Na:} 605.3666\right]$.

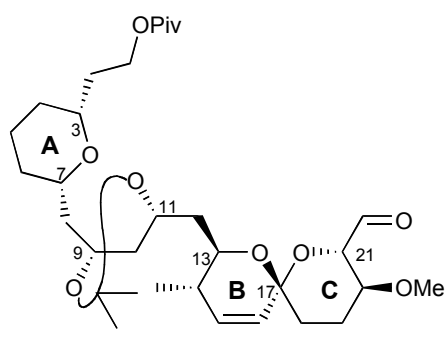

$(+)-24$

Aldehyde (+)-24. To a solution of (+)-S11 $(50 \mathrm{mg}, 0.086 \mathrm{mmol})$ in $\mathrm{CH}_{2} \mathrm{Cl}_{2}(3 \mathrm{~mL})$ at $0{ }^{\circ} \mathrm{C}$ was added $\mathrm{NaHCO}_{3}$ (36 mg, $0.43 \mathrm{mmol}, 3.0$ equiv) and then Dess-Martin periodinane $(0.109 \mathrm{~g}, 0.257$ mmol, 3.0 equiv). The resulting mixture was warmed to ambient temperature and stirred for 1.5 h. A saturated aqueous $\mathrm{Na}_{2} \mathrm{~S}_{2} \mathrm{O}_{3}$ solution $(1 \mathrm{~mL})$, water $(5 \mathrm{~mL})$ and $\mathrm{Et}_{2} \mathrm{O}(10 \mathrm{~mL})$ were then added and the resulting mixture was stirred for $10 \mathrm{~min}$ at ambient temperature. The organic layer was then separated and the aqueous layer was extracted with ether $(2 \times 10 \mathrm{~mL})$. The combined organic layers were washed with brine $(10 \mathrm{~mL})$, dried over $\mathrm{MgSO}_{4}$, filtered, and concentrated in vacuo. Flash chromatography on silica gel, using ethyl acetate-hexanes (1:1) as eluant, provided aldehyde (+)-24 (45 mg, $0.078 \mathrm{mmol}, 90 \%$ yield) as a pale yellow oil: $R_{f} 0.50$ (hexanes/ethyl acetate 1/1); $[\alpha]^{20}{ }_{D}+48\left(c\right.$ 0.5, $\left.\mathrm{C}_{6} \mathrm{H}_{6}\right)$; IR (film) 3035 (w), 2936 (s), $1729(\mathrm{~s}), 1457(\mathrm{~m}), 1379(\mathrm{~m})$, 1285 (m), 1225 (s), 1160 (s), 1017 (s) cm ${ }^{-1} ;{ }^{1} \mathrm{H}$ NMR (500 MHz, $\left.\mathrm{C}_{6} \mathrm{D}_{6}\right) \delta 9.83$ (d, J=1.8 Hz, 1H), $5.57(\mathrm{dd}, J=10.1$ and $2.4 \mathrm{~Hz}, 1 \mathrm{H}), 5.52(\mathrm{dd}, J=10.0$ and $1.4 \mathrm{~Hz}, 1 \mathrm{H}), 4.49(\mathrm{dd}, J=10.0$ and 1.9 $\mathrm{Hz}, 1 \mathrm{H}), 4.42-4.36(\mathrm{~m}, 1 \mathrm{H}), 4.31-4.21(\mathrm{~m}, 3 \mathrm{H}), 3.93(\operatorname{app} \mathrm{t}, J=9.7 \mathrm{~Hz}, 1 \mathrm{H}), 3.55-3.49(\mathrm{~m}, 1 \mathrm{H})$, 3.27-3.21 (m, 1H), 3.10 (ddd, $J=10.4,10.4$ and $4.8 \mathrm{~Hz}, 1 \mathrm{H}), 3.00(\mathrm{~s}, 3 \mathrm{H}), 1.98-1.86(\mathrm{~m}, 3 \mathrm{H})$, 
1.79-1.62 (m, 4H), $1.60(\mathrm{~s}, 3 \mathrm{H}), 1.60-1.40(\mathrm{~m}, 3 \mathrm{H}), 1.50(\mathrm{~s}, 3 \mathrm{H}), 1.35-1.20(\mathrm{~m}, 7 \mathrm{H}), 1.16(\mathrm{~s}, 9 \mathrm{H})$, 1.10-1.01 (m, 2H), $0.76(\mathrm{~d}, J=7.1 \mathrm{~Hz}, 3 \mathrm{H}) ;{ }^{13} \mathrm{C}$ NMR $\left(125 \mathrm{MHz}, \mathrm{C}_{6} \mathrm{D}_{6}\right) \delta 197.7,177.6,134.9$, $128.4,100.3,93.7,76.9,74.5,74.4,74.1,70.6,63.5,62.9,61.5,56.1,43.4,41.5,39.4,38.7$, $36.2,35.0,33.8,32.4,32.0,27.3,26.4,25.7,24.1,24.0,16.8$; high resolution mass spectrum (ESI) $\mathrm{m} / \mathrm{z} 581.3675\left[(\mathrm{M}+\mathrm{H})^{+}\right.$; calcd for $\left.\mathrm{C}_{32} \mathrm{H}_{53} \mathrm{O}_{9}: 581.3690\right]$.

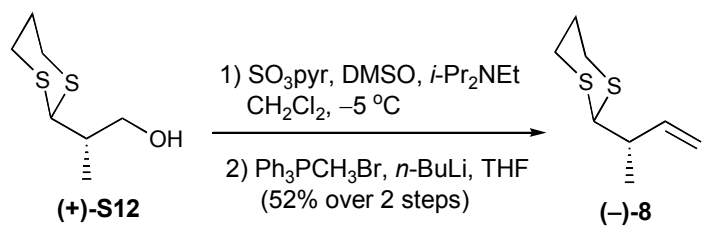

Dithiane (-)-8. To a solution of known alcohol (+)-S12 ${ }^{1}(0.510 \mathrm{~g}, 2.86 \mathrm{mmol})$ in $\mathrm{DMSO}-\mathrm{CH}_{2} \mathrm{Cl}_{2}$ $(3 / 10 \mathrm{~mL})$ at $0{ }^{\circ} \mathrm{C}$ was added DIPEA $\left(2.5 \mathrm{~mL}, 14 \mathrm{mmol}, 5.0\right.$ equiv) followed by $\mathrm{SO}_{3} \mathrm{Pyr}(1.37 \mathrm{~g}$, $8.61 \mathrm{mmol}, 3.0$ equiv). After being stirred for $20 \mathrm{~min}$ at $0{ }^{\circ} \mathrm{C}$, the reaction mixture was poured into a saturated $\mathrm{NH}_{4} \mathrm{Cl}$ aqueous solution $(20 \mathrm{~mL})$ and the resulting mixture was extracted with $\mathrm{Et}_{2} \mathrm{O}$ (30 $\mathrm{mL} \times 2)$. The combined organic layers were washed successively with $1 \mathrm{M} \mathrm{HCl}(20 \mathrm{~mL})$, a saturated $\mathrm{NaHCO}_{3}(20 \mathrm{~mL})$, and brine $(20 \mathrm{~mL})$, dried over $\mathrm{MgSO}_{4}$, filtered and concentrated in vacuo. Without purification, the crude aldehyde was dissolved in THF (10 mL). To a slurry of methyltriphenylphosphonium bromide $\left(3.06 \mathrm{~g}, 8.57 \mathrm{mmol}, 3.0\right.$ equiv) in THF $(20 \mathrm{~mL})$ at $0{ }^{\circ} \mathrm{C}$ was added $n$-BuLi (1.35 M in hexane, $6.4 \mathrm{mmol}, 8.6 \mathrm{mmol}, 3.0$ equiv). After being stirred for $10 \mathrm{~min}$ a $0{ }^{\circ} \mathrm{C}$, the resulting solution was added into the solution of the aldehyde at $0{ }^{\circ} \mathrm{C}$. The reaction mixture was warmed to ambient temperature and stirred for 20 min before a saturated $\mathrm{NH}_{4} \mathrm{Cl}$ aqueous solution $(30 \mathrm{~mL})$ and water $(20 \mathrm{~mL})$ were added. The organic layer was separated and the aqueous layer was extracted with $\mathrm{Et}_{2} \mathrm{O}(50 \mathrm{~mL} \times 2)$. The combined organic layers were washed with brine $(30 \mathrm{~mL})$, dried over $\mathrm{MgSO}_{4}$, filtered and concentrated in vacuo. Flash chromatography on silica gel, using ethyl acetate-hexanes (1:20) as eluant, provided (-)-8 (0.260 g, $0.149 \mathrm{mmol}, 52 \%$ yield over 2 steps) as a colorless oil: $R_{f} 0.50$ (hexanes/ethyl acetate 10/1); $[\alpha]^{20}{ }_{D}-12.4\left(c\right.$ 0.44, $\left.\mathrm{CHCl}_{3}\right)$; IR (film) $3076(\mathrm{~m}), 2963$ (s), 2897 (s), $1640(\mathrm{~m}), 1419$ (s), 1275 (s),

\footnotetext{
${ }^{1}$ Ide, M.; Nakata, M. Bull. Chem. Soc. Jpn. 1999, 72, 2501.
} 
1185 (m), 990 (s), 912 (s) cm ${ }^{-1} ;{ }^{1} \mathrm{H}$ NMR (500 MHz, $\mathrm{CDCl}_{3}$ ) $\delta 5.84$ (ddd, $J=17.5,10.3$ and 7.7 $\mathrm{Hz}, 1 \mathrm{H}), 5.11-5.05(\mathrm{~m}, 2 \mathrm{H}), 4.07(\mathrm{~d}, J=5.5 \mathrm{~Hz}, 1 \mathrm{H}), 2.90-2.80(\mathrm{~m}, 4 \mathrm{H}), 2.57-2.53(\mathrm{~m}, 1 \mathrm{H}), 2.10-$ $2.05(\mathrm{~m}, 1 \mathrm{H}), 1.86-1.78(\mathrm{~m}, 1 \mathrm{H}), 1.19(\mathrm{~d}, J=6.8 \mathrm{~Hz}, 3 \mathrm{H}) ;{ }^{13} \mathrm{C} \mathrm{NMR}\left(125 \mathrm{MHz}, \mathrm{CDCl}_{3}\right) \delta 139.8$, $115.5,54.1,42.9,30.75,30.69,26.0,17.4$; high resolution mass spectrum $(\mathrm{Cl}) \mathrm{m} / \mathrm{z} 175.0614$ $\left[(\mathrm{M}+\mathrm{H})^{+}\right.$; calcd for $\left.\mathrm{C}_{8} \mathrm{H}_{15} \mathrm{~S}_{2}: 175.0615\right]$.

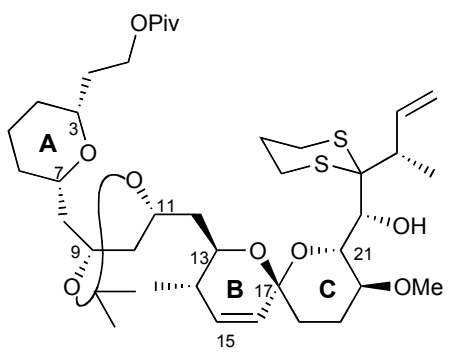

$(+)-26$

Alcohol (+)-26. To a solution of dithiane (-)-8 (22 mg, $0.126 \mathrm{mmol}$. 3.3 equiv) in THF (1.5 mL) at ambient temperature was added n-BuLi (2.15 $\mathrm{M}$ in hexane, $62 \mu \mathrm{L}, 0.13 \mathrm{mmol}, 3.3$ equiv). After being stirred for $5 \mathrm{~min}$ at ambient temperature, the solution was cooled to $-78{ }^{\circ} \mathrm{C}$ and a solution of aldehyde (+)-24 $(22 \mathrm{mg}, 0.038 \mathrm{mmol})$ in THF $(1.5 \mathrm{~mL})$ was added dropwise. After $3 \mathrm{~min}$ at -78 ${ }^{\circ} \mathrm{C}$, a saturated $\mathrm{NH}_{4} \mathrm{Cl}$ aqueous solution $(3 \mathrm{~mL})$ and water $(3 \mathrm{~mL})$ were added and the resulting mixture was warmed to ambient temperature. The mixture was then extracted with $\mathrm{Et}_{2} \mathrm{O}(8 \mathrm{~mL} X$ 3) and the combined organic layers were washed with brine and dried over $\mathrm{MgSO}_{4}$. After removal of solvents, the residue was dissolved in $\mathrm{EtOH}(2 \mathrm{~mL})$ and the resulting solution was cooled to $0{ }^{\circ} \mathrm{C}$. Excess $\mathrm{NaBH}_{4}$ (ca. $15 \mathrm{mg}, 0.40 \mathrm{mmmol}, 10$ equiv) was then added and stirred for 2 min at $0{ }^{\circ} \mathrm{C}$ before a saturated $\mathrm{NH}_{4} \mathrm{Cl}$ solution $(10 \mathrm{~mL})$ was added. The resulting mixture was extracted with $\mathrm{Et}_{2} \mathrm{O}(10 \mathrm{~mL} \times 3)$. The combined organic layers were washed with brine $(10 \mathrm{~mL})$, dried over $\mathrm{MgSO}_{4}$, filtered, and concentrated in vacuo. Flash chromatography on silica gel, using ethyl acetate-hexanes $(1: 3 \rightarrow 1: 1)$ as eluant, provided the desired alcohol $(+)-26(2.1 \mathrm{mg}$, $2.8 \mu \mathrm{mol}, 7.5 \%$ yield $)$ as a white foam, epimer (+)-25 (14 mg, $19 \mathrm{mmol}, 49 \%$ yield) as a white foam, and alcohol (+)-S11 (2.4 mg, $4.2 \mu \mathrm{mol}, 11 \%$ yield). For $(+)-26: R_{f} 0.50$ (hexanes/ethyl

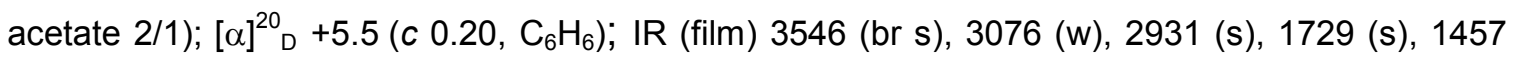


(m), $1377(\mathrm{~m}), 1225(\mathrm{~m}), 1158$ (s), $1103(\mathrm{~s}) \mathrm{cm}^{-1} ;{ }^{1} \mathrm{H}$ NMR $\left(500 \mathrm{MHz}, \mathrm{C}_{6} \mathrm{D}_{6}\right) \delta 6.68$ (ddd, $J=17.2$, 10.0 and $9.3 \mathrm{~Hz}, 1 \mathrm{H}), 5.45(\mathrm{dd}, J=10.0$ and $2.0 \mathrm{~Hz}, 1 \mathrm{H}), 5.42(\mathrm{dd}, J=10.1$ and $1.1 \mathrm{~Hz}, 1 \mathrm{H}), 5.27$ (dd, $J=17.2$ and $1.9 \mathrm{~Hz}, 1 \mathrm{H}), 5.01(\mathrm{dd}, J=10.2$ and $2.1 \mathrm{~Hz}, 1 \mathrm{H}), 4.71(\mathrm{~d}, J=10.5 \mathrm{~Hz}, 1 \mathrm{H}), 4.66$ (d, $J=9.5 \mathrm{~Hz}, 1 \mathrm{H}), 4.61-4.55(\mathrm{~m}, 1 \mathrm{H}), 4.43-4.38(\mathrm{~m}, 1 \mathrm{H}), 4.36-4.26(\mathrm{~m}, 2 \mathrm{H}), 3.99(\mathrm{app} \mathrm{td}, J=9.7$ and $2.3 \mathrm{~Hz}, 1 \mathrm{H}), 3.63-3.58(\mathrm{~m}, 1 \mathrm{H}), 3.41(\mathrm{ddd}, J=10.9,10.2$ and $5.0 \mathrm{~Hz}, 1 \mathrm{H}), 3.35-3.27(\mathrm{~m}, 2 \mathrm{H})$, $3.15(\mathrm{~s}, 3 \mathrm{H}), 3.02(\mathrm{~d}, J=10.5 \mathrm{~Hz}, 1 \mathrm{H}), 3.02-2.98(\mathrm{~m}, 1 \mathrm{H}), 2.68(\mathrm{ddd}, J=14.0,8.8$ and $3.9 \mathrm{~Hz}$, 1H), 2.47-2.42 (m, 2H), 2.29-2.21 (m, 2H), $1.99(\mathrm{ddd}, J=13.5,8.1$ and $5.6 \mathrm{~Hz}, 1 \mathrm{H}), 1.93-1.78(\mathrm{~m}$, 4H), 1.77-1.64 (m, 5H), $1.62(\mathrm{~d}, J=7.0 \mathrm{~Hz}, 3 \mathrm{H}), 1.59(\mathrm{~s}, 6 \mathrm{H}), 1.58-1.50(\mathrm{~m}, 2 \mathrm{H}), 1.42-1.21(\mathrm{~m}$, $5 \mathrm{H}), 1.18(\mathrm{~s}, 9 \mathrm{H}), 1.16-1.03(\mathrm{~m}, 2 \mathrm{H}), 0.76(\mathrm{~d}, J=7.1 \mathrm{~Hz}, 3 \mathrm{H}) ;{ }^{13} \mathrm{C}$ NMR $\left(125 \mathrm{MHz}, \mathrm{C}_{6} \mathrm{D}_{6}\right) \delta 177.6$, 143.0, 134.1, 128.7, 117.1, 100.2, 93.9, 75.0, 74.5, 74.3, 74.0, 72.5, 71.7, 67.0, 63.1, 62.5, 61.6, $55.8,45.9,43.7,41.4,38.7,36.2,35.0,34.1,32.4,32.0,27.3,26.3,25.9,25.7,25.6,24.9,24.0$, 23.5, 18.3, 17.2; high resolution mass spectrum $(\mathrm{ESI}) \mathrm{m} / \mathrm{z} 777.4024\left[(\mathrm{M}+\mathrm{Na})^{+}\right.$; calcd for $\mathrm{C}_{40} \mathrm{H}_{66} \mathrm{O}_{9} \mathrm{NaS}_{2}:$ 777.4046].

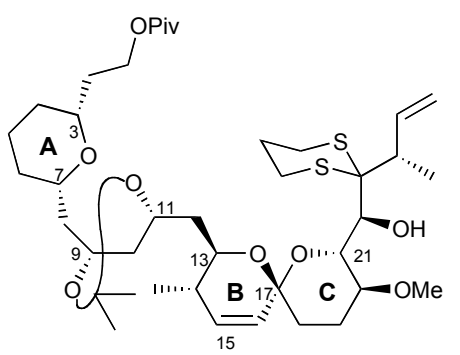

$(+)-25$

For (+)-25: $\mathrm{R}_{\mathrm{f}} 0.60$ (hexanes/ethyl acetate 2/1); $[\alpha]^{20}{ }_{\mathrm{D}}+12\left(c \quad 0.55, \mathrm{C}_{6} \mathrm{H}_{6}\right)$; IR (film) 3444 (br s), 3078 (w), 2927 (s), 1729 (s), 1457 (m), 1377 (m), $1226(\mathrm{~m}), 1160$ (s), $802(\mathrm{~m}) \mathrm{cm}^{-1} ;{ }^{1} \mathrm{H}$ NMR (500 $\left.\mathrm{MHz}, \mathrm{C}_{6} \mathrm{D}_{6}\right) \delta 6.48(\mathrm{ddd}, J=17.1,10.2$ and $8.8 \mathrm{~Hz}, 1 \mathrm{H}), 5.50(\mathrm{dd}, J=10.0$ and $2.4 \mathrm{~Hz}, 1 \mathrm{H}), 5.45$ (dd, $J=10.0$ and $2.8 \mathrm{~Hz}, 1 \mathrm{H}$ ), $5.33(\mathrm{dd}, J=17.0$ and $1.9 \mathrm{~Hz}, 1 \mathrm{H}), 5.17(\mathrm{dd}, J=10.2$ and $2.1 \mathrm{~Hz}$, 1H), 4.64-4.60 (m, 1H), $4.57(\mathrm{dd}, J=5.0$ and $2.8 \mathrm{~Hz}, 1 \mathrm{H}), 4.49(\mathrm{dd}, J=9.4$ and $5.1 \mathrm{~Hz}, 1 \mathrm{H}), 4.44-$ $4.38(\mathrm{~m}, 1 \mathrm{H}), 4.36-4.27(\mathrm{~m}, 2 \mathrm{H}), 4.11(\mathrm{app} \mathrm{td}, J=9.6$ and $2.4 \mathrm{~Hz}, 1 \mathrm{H}), 3.90(\mathrm{br} \mathrm{s}, 1 \mathrm{H}), 3.47-3.36$ $(\mathrm{m}, 2 \mathrm{H}), 3.34-3.27(\mathrm{~m}, 2 \mathrm{H}), 2.99(\mathrm{~s}, 3 \mathrm{H}), 2.99-2.93(\mathrm{~m}, 1 \mathrm{H}), 2.36-2.17(\mathrm{~m}, 4 \mathrm{H}), 1.98(\mathrm{ddd}, J=$ 13.6, 8.4 and $5.7 \mathrm{~Hz}, 1 \mathrm{H}), 1.92-1.80(\mathrm{~m}, 3 \mathrm{H}), 1.79-1.70(\mathrm{~m}, 5 \mathrm{H}), 1.71(\mathrm{~d}, J=6.8 \mathrm{~Hz}, 3 \mathrm{H}), 1.70-$ $1.63(\mathrm{~m}, 2 \mathrm{H}), 1.62(\mathrm{~s}, 3 \mathrm{H}), 1.61(\mathrm{~s}, 3 \mathrm{H}), 1.58-1.52(\mathrm{~m}, 2 \mathrm{H}), 1.40-1.34(\mathrm{~m}, 2 \mathrm{H}), 1.30-1.21(\mathrm{~m}, 2 \mathrm{H})$, 
$1.18(\mathrm{~s}, 9 \mathrm{H}), 1.15-1.03(\mathrm{~m}, 2 \mathrm{H}), 0.78(\mathrm{~d}, J=7.1 \mathrm{~Hz}, 3 \mathrm{H}) ;{ }^{13} \mathrm{C}$ NMR $\left(125 \mathrm{MHz}, \mathrm{C}_{6} \mathrm{D}_{6}\right) \delta 177.6$, $141.2,134.2,128.7,115.7,100.2,93.3,80.5,77.9,74.5,74.3,73.8,73.2,66.4,63.1,61.6,61.4$, $54.9,44.8,43.8,42.2,41.4,38.7,36.2,35.2,34.0,32.4,32.1,27.6,27.3,26.5,26.2,25.7,24.9$, 24.0, 23.7, 18.5, 17.5; high resolution mass spectrum (ESI) $\mathrm{m} / \mathrm{z} 777.4083\left[(\mathrm{M}+\mathrm{Na})^{+}\right.$; calcd for $\mathrm{C}_{40} \mathrm{H}_{66} \mathrm{O}_{9} \mathrm{NaS}_{2}:$ 777.4046].

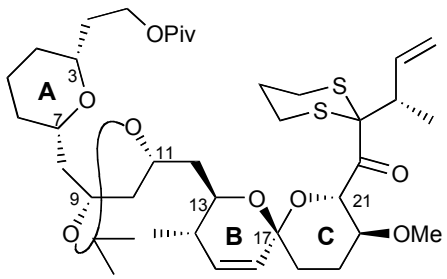

$(+)-S 13$

Ketone (+)-S13. To a solution of $(+)-25(23 \mathrm{mg}, 0.030 \mathrm{mmol})$ in $\mathrm{CH}_{2} \mathrm{Cl}_{2}(3.5 \mathrm{~mL})$ at $0{ }^{\circ} \mathrm{C}$ was added $\mathrm{NaHCO}_{3}(13 \mathrm{mg}, 0.15 \mathrm{mmol}, 5.0$ equiv) and then Dess-Martin periodinane (39 mg, 0.092 $\mathrm{mmol}, 3.0$ equiv). After being stirred for $30 \mathrm{~min}$ at $0{ }^{\circ} \mathrm{C}$, the reaction mixture was loaded onto a silica gel column. Flash chromatography on silica gel, using ethyl acetate-hexanes (1:4) as eluant, provided ketone (+)-S13 $\left(22 \mathrm{mg}, 0.029 \mathrm{mmol}, 96 \%\right.$ yield) as a colorless oil: $R_{\mathrm{f}} 0.55$ (hexanes/ethyl acetate 2/1); $[\alpha]^{20}{ }_{D}+26\left(c 0.66, \mathrm{C}_{6} \mathrm{H}_{6}\right)$; IR (film) $3082(\mathrm{w}), 29832(\mathrm{~s}), 1729$ (s), $1460(\mathrm{~m}), 1375(\mathrm{~m}), 1225(\mathrm{~m}), 1159(\mathrm{~s}), 927(\mathrm{~m}) \mathrm{cm}^{-1} ;{ }^{1} \mathrm{H}$ NMR $\left(500 \mathrm{MHz}, \mathrm{C}_{6} \mathrm{D}_{6}\right) \delta 6.44(\mathrm{ddd}, J=$ 17.1, 10.1 and $8.4 \mathrm{~Hz}, 1 \mathrm{H}$ ), $5.56(\mathrm{~d}, J=17.2 \mathrm{~Hz}, 1 \mathrm{H}), 5.53(\mathrm{dd}, J=10.0$ and $2.5 \mathrm{~Hz}, 1 \mathrm{H}$ ), 5.42 (dd, $J=10.0$ and $1.8 \mathrm{~Hz}, 1 \mathrm{H}$ ), $5.24(\mathrm{dd}, J=10.6$ and $1.2 \mathrm{~Hz}, 1 \mathrm{H}), 5.17(\mathrm{~d}, J=9.4 \mathrm{~Hz}, 1 \mathrm{H}$ ), 4.63$4.59(\mathrm{~m}, 1 \mathrm{H}), 4.41(\mathrm{dddd}, J=14.1,9.3,5.6$ and $5.6 \mathrm{~Hz}, 1 \mathrm{H}), 4.35-4.27(\mathrm{~m}, 2 \mathrm{H}), 3.84(\mathrm{app} \mathrm{td}, J=$ 9.8 and $2.4 \mathrm{~Hz}, 1 \mathrm{H}), 3.72(\mathrm{ddd}, J=11.0,9.6$ and $5.1 \mathrm{~Hz}, 1 \mathrm{H}), 3.63-3.58(\mathrm{~m}, 1 \mathrm{H}), 3.31-3.01(\mathrm{~m}$, 1H), 3.22 (app t, $J=7.4 \mathrm{~Hz}, 1 \mathrm{H}), 3.08-3.01(\mathrm{~m}, 2 \mathrm{H}), 2.96(\mathrm{~s}, 3 \mathrm{H}), 2.28-2.16(\mathrm{~m}, 4 \mathrm{H}), 2.01$ (ddd, $J$ $=13.4,8.8$ and $5.9 \mathrm{~Hz}, 1 \mathrm{H}), 1.89-1.65(\mathrm{~m}, 9 \mathrm{H}), 1.63(\mathrm{~s}, 3 \mathrm{H}), 1.60(\mathrm{~s}, 3 \mathrm{H}), 1.60-1.46(\mathrm{~m}, 3 \mathrm{H})$, 1.36-1.22 (m, 4H), $1.32(\mathrm{~d}, J=6.8 \mathrm{~Hz}, 3 \mathrm{H}), 1.18(\mathrm{~s}, 9 \mathrm{H}), 1.14-0.84(\mathrm{~m}, 2 \mathrm{H}), 0.69(\mathrm{~d}, J=7.1 \mathrm{~Hz}$, $3 \mathrm{H}) ;{ }^{13} \mathrm{C}$ NMR $\left(125 \mathrm{MHz}, \mathrm{C}_{6} \mathrm{D}_{6}\right) \delta 199.5,177.6,138.6,134.5,128.5,117.5,100.3,94.5,76.1$, $74.5,74.2,73.9,71.0,69.3,66.7,63.1,61.6,55.7,44.0,43.0,42.1,42.0,38.7,36.2,35.0,33.8$, 
$32.4,32.0,28.6,27.4,27.3,26.4,25.6,25.1,24.0,23.4,17.5,16.7$; high resolution mass spectrum (ESI) m/z $775.3904\left[(\mathrm{M}+\mathrm{Na})^{+} ;\right.$calcd for $\mathrm{C}_{40} \mathrm{H}_{64} \mathrm{O}_{9} \mathrm{NaS}_{2}:$ 775.3889].

Keotone (+)-S13 $\rightarrow$ Alcohol (+)-26. To a solution of ketone $(+)-S 13(5.0 \mathrm{mg}, 6.6 \mu \mathrm{mol})$ in $\mathrm{CH}_{2} \mathrm{Cl}_{2}$ at $-78{ }^{\circ} \mathrm{C}$ was added DIBAL-H $(1.0 \mathrm{M}$ in hexane, $0.08 \mathrm{~mL}, 0.08 \mathrm{mmol}, 12$ equiv) over $2 \mathrm{~h}$ in 4 portions $\left(0.02 \mathrm{~mL}\right.$ each time). The resulting solution was stirred for additional $1 \mathrm{~h}$ at $-78{ }^{\circ} \mathrm{C}$ and the excess DIBAL-H was then quenched with $\mathrm{MeOH}(0.1 \mathrm{~mL})$. After a saturated Rochelle's salt solution $(2 \mathrm{~mL})$ was added, the resultant mixture was warmed to ambient temperature and stirred until the mixture became clear $(1 \mathrm{~h})$. The mixture was extracted with $\mathrm{CH}_{2} \mathrm{Cl}_{2}(5 \mathrm{~mL} \times 3)$. The combined organic layers were washed with brine, dried over $\mathrm{MgSO}_{4}$, filtered, and concentrated in vacuo. The residue was further dried by azeotropic removal of water with benzene (2 times). The crude product was dissolved in $\mathrm{CH}_{2} \mathrm{Cl}_{2}(2 \mathrm{~mL})$ and DMAP (24 mg, 0.20 mmol, 30 equiv) was added at ambient temperature. The solution was cooled to $-45{ }^{\circ} \mathrm{C}$ and $\mathrm{PivCl}$ ( $0.016 \mathrm{~mL}, 0.13 \mathrm{mmol}, 20$ equiv) was added. The resultant mixture was warmed to $-20{ }^{\circ} \mathrm{C}$ over 30 min and a saturated $\mathrm{NaHCO}_{3}$ solution $(5 \mathrm{~mL})$ was added. The mixture was warmed to ambient temperature and extracted with $\mathrm{Et}_{2} \mathrm{O}(5 \mathrm{~mL} \times 3)$. The combined organic layers were washed with brine $(5 \mathrm{~mL})$, dried over $\mathrm{MgSO}_{4}$, filtered, and concentrated in vacuo. Flash chromatography on silica gel, using ethyl acetate-hexanes $(1: 4 \rightarrow 1: 2)$ as eluant, provided alcohol (+)-26 (2.5 mg, $3.3 \mu \mathrm{mol}, 50 \%$ yield over 2 steps, 78\% BORSM) as a colorless oil and ketone (+)S13 (1.8 mg, $2.4 \mu \mathrm{mol}, 36 \%$ recovered).

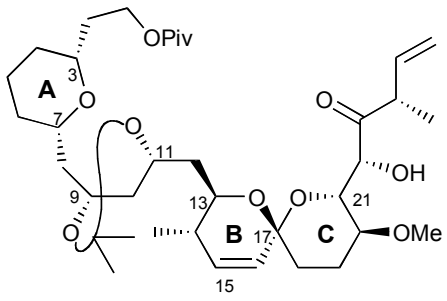

$(+)-27$

Ketone (+)-27. A solution of dithiane (+)-26 (3.5 mg, $4.6 \mu \mathrm{mol})$ in $\mathrm{CH}_{3} \mathrm{CN}(1 \mathrm{~mL})$ was added to a stirred solution of $\mathrm{NCS}$ (6.2 mg, $0.046 \mathrm{mmol}, 10$ equiv), $\mathrm{AgNO}_{3}(12 \mathrm{mg}, 0.071 \mathrm{mmol}, 15$ equiv) 
and 2,6-lutidine $\left(0.017 \mathrm{~mL}, 0.094 \mathrm{mmol}, 20\right.$ equiv) in aqueous $\mathrm{CH}_{3} \mathrm{CN}(1.4 / 0.6 \mathrm{~mL})$ at $0{ }^{\circ} \mathrm{C}$. After being stirred for $5 \mathrm{~min}$ at $0{ }^{\circ} \mathrm{C}$, the reaction mixture was diluted with $\mathrm{Et}_{2} \mathrm{O}(10 \mathrm{~mL})$ and washed with a saturated $\mathrm{NaHSO}_{3}$ solution $(2 \mathrm{~mL})$, a saturated $\mathrm{NaHCO}_{3}$ solution $(2 \mathrm{~mL})$ and brine $(2 \mathrm{~mL})$. The resulting organic layer was then dried over $\mathrm{MgSO}_{4}$, filtered, and concentrated in vacuo. Flash chromatography on silica gel, using ethyl acetate-hexanes (1:2) as eluant, provided ketone $(+)-27\left(1.7 \mathrm{mg}, 2.6 \mu \mathrm{mol}, 56 \%\right.$ yield) as a colorless oil: $\mathrm{R}_{\mathrm{f}} 0.30$ (hexanes/ethyl acetate $\left.2 / 1\right) ;[\alpha]^{20}$ +18 (c 0.13, $\left.\mathrm{C}_{6} \mathrm{H}_{6}\right) ;$ IR (film) 3454 (br m), 3076 (w), 2927 (s), 2856 (s), 1729 (s), 1457 (m), 1378 (m), 1286 (m), 1159 (s), 1012 (s) cm ${ }^{-1} ;{ }^{1} \mathrm{H}$ NMR (500 MHz, $\left.\mathrm{C}_{6} \mathrm{D}_{6}\right) \delta 6.21$ (ddd, $J=17.6,10.5$ and 8.5 Hz, 1H), $5.43(\mathrm{dd}, J=10.0$ and $2.1 \mathrm{~Hz}, 1 \mathrm{H}), 5.41(\mathrm{dd}, J=10.0$ and $1.4 \mathrm{~Hz}, 1 \mathrm{H}), 5.24(\mathrm{~d}, J=$ $17.4 \mathrm{~Hz}, 1 \mathrm{H}$ ), $5.04(\mathrm{~d}, J=10.4 \mathrm{~Hz}, 1 \mathrm{H}), 4.77$ (dd, $J=7.8$ and $2.3 \mathrm{~Hz}, 1 \mathrm{H}$ ), 4.51 (app qd, $J=7.8$ and $4.5 \mathrm{~Hz}, 1 \mathrm{H}), 4.39(\mathrm{dd}, J=9.5$ and $2.3 \mathrm{~Hz}, 1 \mathrm{H}), 4.37-4.31(\mathrm{~m}, 1 \mathrm{H}), 4.31-4.25(\mathrm{~m}, 2 \mathrm{H}), 3.82$ (app quin, $J=7.3 \mathrm{~Hz}, 1 \mathrm{H}$ ), $3.71(\operatorname{app} \mathrm{td}, J=9.9$ and $2.5 \mathrm{~Hz}, 1 \mathrm{H}), 3.60-3.53(\mathrm{~m}, 2 \mathrm{H}), 3.51(\mathrm{~d}, J=$ 7.8 Hz, 1H), 3.30-3.26 (m, 1H), $3.18(\mathrm{~s}, 3 \mathrm{H}), 2.02(\mathrm{ddd}, J=14.4,7.7$ and $2.6 \mathrm{~Hz}, 1 \mathrm{H}), 1.98-1.92$ $(\mathrm{m}, 1 \mathrm{H}), 1.84-1.76(\mathrm{~m}, 3 \mathrm{H}), 1.75-1.70(\mathrm{~m}, 1 \mathrm{H}), 1.73(\mathrm{~s}, 3 \mathrm{H}), 1.69-1.61(\mathrm{~m}, 3 \mathrm{H}), 1.59(\mathrm{~s}, 3 \mathrm{H}), 1.58-$ $1.50(\mathrm{~m}, 3 \mathrm{H}), 1.41(\mathrm{app} t \mathrm{~d}, J=13.4$, and $4.0 \mathrm{~Hz}, 1 \mathrm{H}), 1.34-1.21(\mathrm{~m}, 4 \mathrm{H}), 1.17(\mathrm{~s}, 9 \mathrm{H}), 1.09(\mathrm{~d}, J=$ 7.1 Hz, 3H), 1.13-1.00 (m, 2H), $0.76(\mathrm{~d}, J=7.3 \mathrm{~Hz}, 3 \mathrm{H}) ;{ }^{13} \mathrm{C} N M R\left(125 \mathrm{MHz}, \mathrm{C}_{6} \mathrm{D}_{6}\right) \delta 211.6$, 177.6, 137.6, 134.0, 127.5, 116.2, 100.4, 93.6, 74.7, 74.5, 74.3, 74.2, 74.1, 70.6, 64.1, 63.0 ,61.6, $56.1,45.5,43.5,42.5,40.5,38.7,36.2,35.5,34.1,32.4,32.0,27.3,26.4,26.0,24.0,23.6,18.2$, 17.1; high resolution mass spectrum $(\mathrm{ESI}) \mathrm{m} / \mathrm{z} 687.4099\left[(\mathrm{M}+\mathrm{Na})^{+}\right.$; calcd for $\mathrm{C}_{37} \mathrm{H}_{60} \mathrm{O}_{10} \mathrm{Na}$ : 687.4084].

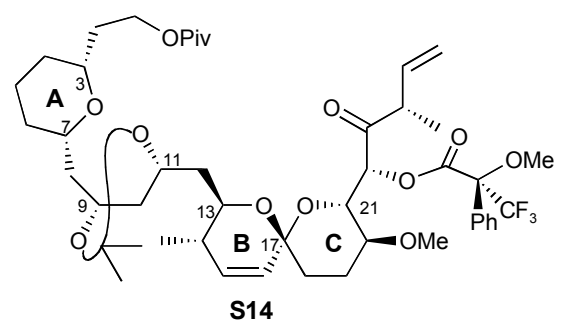

(S)-MTPA ester S14. To a solution of (+)-27 $(0.7 \mathrm{mg}, 1.1 \mu \mathrm{mol})$ in $\mathrm{CH}_{2} \mathrm{Cl}_{2}(0.3 \mathrm{~mL})$ at $0{ }^{\circ} \mathrm{C}$ was added DMAP (5 mg, $0.041 \mathrm{mmol}, 39$ equiv) followed by $(R)-(-)-M T P A$ chloride $(5 \mu \mathrm{L}, 0.027$ 
mmol, 26 equiv). The resulting mixture was warmed to ambient temperature and stirred for 15 min. Without work-up, the reaction mixture was loaded on a silica gel column. Flash chromatography on silica gel, using ethyl acetate-hexanes $(1: 4 \rightarrow 1: 3)$ as eluant, provided (S)MTPA ester $\mathbf{S} 14$ (0.8 mg, $0.9 \mu \mathrm{mol}, 90 \%$ yield) as a colorless oil: $\mathrm{R}_{\mathrm{f}} 0.30$ (hexanes/ethyl acetate 4/1); ${ }^{1} \mathrm{H}$ NMR (500 MHz, $\left.\mathrm{CDCl}_{3}\right) \delta$ 7.65-7.62 (m, 2H), 7.47-7.41 (m, 3H), 5.86 (ddd, $J=17.6,10.4$ and $7.4 \mathrm{~Hz}, 1 \mathrm{H}), 5.72(\mathrm{~d}, J=2.6 \mathrm{~Hz}, 1 \mathrm{H}), 5.58(\mathrm{dd}, J=10.0$ and $1.9 \mathrm{~Hz}, 1 \mathrm{H}), 5.42(\mathrm{dd}, J=10.0$ and $2.6 \mathrm{~Hz}, 1 \mathrm{H}), 5.04(\mathrm{~d}, J=17.5 \mathrm{~Hz}, 1 \mathrm{H}), 5.01(\mathrm{~d}, J=10.8 \mathrm{~Hz}, 1 \mathrm{H}), 4.28(\mathrm{dd}, J=9.6$ and $2.7 \mathrm{~Hz}$, 1H), 4.26-4.08 (m, 4H), $3.58(\mathrm{~s}, 3 \mathrm{H}), 3.53-3.48(\mathrm{~m}, 1 \mathrm{H}), 3.49$ (app quin, $J=7.1 \mathrm{~Hz}, 1 \mathrm{H}), 3.42$ (app td, $J=9.6$ and $2.6 \mathrm{~Hz}, 1 \mathrm{H}), 3.42-3.38(\mathrm{~m}, 1 \mathrm{H}), 3.25(\mathrm{~s}, 3 \mathrm{H}), 3.20(\mathrm{app} t \mathrm{td}, J=10.4$ and $4.8 \mathrm{~Hz}$, 1H), 2.09-2.04 (m, 1H), 1.98 (ddd. $J=14.4,6.8$ and $2.7 \mathrm{~Hz}, 1 \mathrm{H}), 1.96-1.90(\mathrm{~m}, 1 \mathrm{H}), 1.84-1.71(\mathrm{~m}$, $6 \mathrm{H}), 1.65(\mathrm{ddd}, J=13.5,8.3$ and $5.6 \mathrm{~Hz}, 1 \mathrm{H}), 1.59-1.50(\mathrm{~m}, 9 \mathrm{H}), 1.47(\mathrm{~s}, 3 \mathrm{H}), 1.37(\mathrm{~s}, 3 \mathrm{H}), 1.22$ $(\mathrm{d}, J=7.1 \mathrm{~Hz}, 3 \mathrm{H}), 1.19(\mathrm{~s}, 9 \mathrm{H}), 0.88(\mathrm{~d}, J=7.1 \mathrm{~Hz}, 3 \mathrm{H})$; high resolution mass spectrum (ESI) $\mathrm{m} / \mathrm{z} 903.4478\left[(\mathrm{M}+\mathrm{Na})^{+}\right.$; calcd for $\mathrm{C}_{47} \mathrm{H}_{67} \mathrm{O}_{12} \mathrm{~F}_{3} \mathrm{Na}$ : 903.4482].

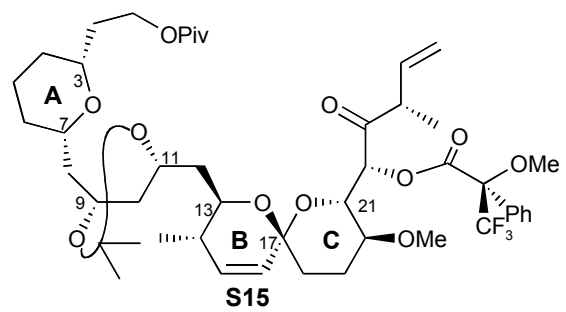

(R)-MTPA ester S15. To a solution of (+)-27 $(0.7 \mathrm{mg}, 1.1 \mu \mathrm{mol})$ in $\mathrm{CH}_{2} \mathrm{Cl}_{2}(0.3 \mathrm{~mL})$ at $0{ }^{\circ} \mathrm{C}$ was added DMAP (5 mg, $0.041 \mathrm{mmol}, 39$ equiv) and then (S)-(+)-MTPA chloride $(5 \mu \mathrm{L}, 0.027 \mathrm{mmol}$, 26 equiv). The resulting mixture was warmed to ambient temperature and stirred for $15 \mathrm{~min}$. Without work-up, the reaction mixture was loaded on a silica gel column. Flash chromatography on silica gel, using ethyl acetate-hexanes $(1: 4 \rightarrow 1: 3)$ as eluant, provided $(R)$-MTPA ester S15 (0.8 mg, $0.9 \mu \mathrm{mol}, 90 \%$ yield) as a colorless oil: $\mathrm{R}_{\mathrm{f}} 0.30$ (hexanes/ethyl acetate $\left.4 / 1\right) ;{ }^{1} \mathrm{H}$ NMR (500 $\left.\mathrm{MHz}, \mathrm{CDCl}_{3}\right) \delta$ 7.73-7.71 (m, 2H), 7.44-7.40 (m, 3H), $5.95(\mathrm{ddd}, J=17.5,10.3$ and $7.2 \mathrm{~Hz}, 1 \mathrm{H})$, $5.65(\mathrm{~d}, J=2.6 \mathrm{~Hz}, 1 \mathrm{H}), 5.57(\mathrm{dd}, J=10.0$ and $1.8 \mathrm{~Hz}, 1 \mathrm{H}), 5.40(\mathrm{dd}, J=10.0$ and $2.7 \mathrm{~Hz}, 1 \mathrm{H})$, $5.11(\mathrm{~d}, J=17.4 \mathrm{~Hz}, 1 \mathrm{H}), 5.07$ (d, $J=10.4 \mathrm{~Hz}, 1 \mathrm{H}$ ), 4.25 (dd, $J=9.5$ and $2.7 \mathrm{~Hz}, 1 \mathrm{H}), 4.23-4.09$ 
(m, 4H), $3.68(\mathrm{~s}, 3 \mathrm{H}), 3.51(\operatorname{app}$ quin, $J=7.1 \mathrm{~Hz}, 1 \mathrm{H}), 3.52-3.47(\mathrm{~m}, 1 \mathrm{H}), 3.42($ app td, $J=9.6$ and $2.5 \mathrm{~Hz}, 1 \mathrm{H}), 3.42-3.37(\mathrm{~m}, 1 \mathrm{H}), 3.06-3.00(\mathrm{~m}, 1 \mathrm{H}), 3.03(\mathrm{~s}, 3 \mathrm{H}), 2.05-1.96(\mathrm{~m}, 2 \mathrm{H}), 1.94-1.90(\mathrm{~m}$, 1H), $1.85-1.68(\mathrm{~m}, 6 \mathrm{H}), 1.63(\mathrm{ddd} . J=13.4,8.4$ and $5.6 \mathrm{~Hz}, 1 \mathrm{H}), 1.57-1.48(\mathrm{~m}, 9 \mathrm{H}), 1.45(\mathrm{~s}, 3 \mathrm{H})$, $1.37(\mathrm{~s}, 3 \mathrm{H}), 1.28(\mathrm{~d}, J=7.2 \mathrm{~Hz}, 3 \mathrm{H}), 1.19(\mathrm{~s}, 9 \mathrm{H}), 0.88(\mathrm{~d}, J=7.1 \mathrm{~Hz}, 3 \mathrm{H})$; high resolution mass spectrum (ESI) m/z $903.4457\left[(\mathrm{M}+\mathrm{Na})^{+}\right.$; calcd for $\mathrm{C}_{47} \mathrm{H}_{67} \mathrm{O}_{12} \mathrm{~F}_{3} \mathrm{Na:}$ 903.4482].

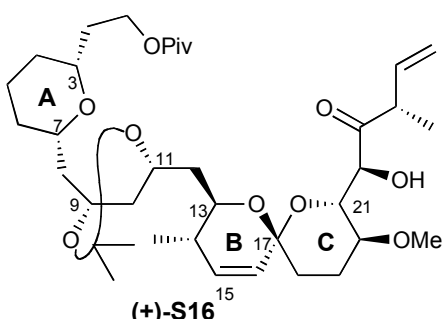

Ketone (+)-S16. A solution of dithiane (+)-25 (2.3 mg, 3.0 $\mu \mathrm{mol})$ in $\mathrm{CH}_{3} \mathrm{CN}(0.5 \mathrm{~mL})$ was added to a stirred solution of NCS (5.0 mg, $0.037 \mathrm{mmol}, 12$ equiv), $\mathrm{AgNO}_{3}(10 \mathrm{mg}, 0.059 \mathrm{mmol}, 19$ equiv) and 2,6-lutidine $\left(0.015 \mathrm{~mL}, 0.13 \mathrm{mmol}, 43\right.$ equiv) in aqueous $\mathrm{CH}_{3} \mathrm{CN}(0.5 / 0.25 \mathrm{~mL})$ at $0{ }^{\circ} \mathrm{C}$. The reaction solution was stirred for $10 \mathrm{~min}$ at $0{ }^{\circ} \mathrm{C}$ and a saturated $\mathrm{NaHSO}_{3}(1 \mathrm{~mL})$ was added. The resulting mixture was diluted with $\mathrm{Et}_{2} \mathrm{O}(5 \mathrm{~mL})$ and water $(3 \mathrm{~mL})$. The organic layer was separated and the aqueous layer was extracted with $\mathrm{Et}_{2} \mathrm{O}(5 \mathrm{~mL} X 2)$. The combined organic layers were washed with a saturated $\mathrm{NaHCO}_{3}$ solution $(5 \mathrm{~mL})$ and brine $(5 \mathrm{~mL})$, dried over $\mathrm{MgSO}_{4}$, filtered, and concentrated in vacuo. Flash chromatography on silica gel, using ethyl acetate-hexanes (1:2) as eluant, provided ketone (+)-S16 (1.1 mg, $1.7 \mu \mathrm{mol}, 54 \%$ yield) as a colorless oil: $\mathrm{R}_{\mathrm{f}} 0.25$ (hexanes/ethyl acetate 3/1); $[\alpha]^{20}{ }_{\mathrm{D}}+95\left(\mathrm{c} 0.10, \mathrm{C}_{6} \mathrm{H}_{6}\right)$; IR (film) 3458 (br s), 3084 (w), 3038 (w), 2934 (s), 2871 (s), 1729 (s), 1458 (m), 1379 (m), 1226 (m), 1159 (s), 1017 (m), $951(\mathrm{~m}) \mathrm{cm}^{-1} ;{ }^{1} \mathrm{H}$ NMR $\left(500 \mathrm{MHz}, \mathrm{C}_{6} \mathrm{D}_{6}\right) \delta 5.75$ (ddd, $J=17.8,10.1$ and $7.8 \mathrm{~Hz}, 1 \mathrm{H}$ ), 5.58 (dd, $J=10.0$ and $2.5 \mathrm{~Hz}, 1 \mathrm{H}), 5.47(\mathrm{dd}, J=10.0$ and $1.8 \mathrm{~Hz}, 1 \mathrm{H}), 5.14(\mathrm{~d}, J=17.2 \mathrm{~Hz}, 1 \mathrm{H}), 4.92$ (d, $J=10.3 \mathrm{~Hz}, 1 \mathrm{H}$ ), 4.70 (dd, $J=4.2$ and $2.5 \mathrm{~Hz}, 1 \mathrm{H}), 4.36-4.24(\mathrm{~m}, 5 \mathrm{H}), 3.84($ app td, $J=9.5$ and $2.1 \mathrm{~Hz}, 1 \mathrm{H}), 3.81(\mathrm{~d}, J=4.5 \mathrm{~Hz}, 1 \mathrm{H}), 3.73(\operatorname{app}$ quin, $J=7.2 \mathrm{~Hz}, 1 \mathrm{H}), 3.57-3.53(\mathrm{~m}, 1 \mathrm{H}), 3.33$ (ddd, $J=10.6,10.6$ and $4.5 \mathrm{~Hz}, 1 \mathrm{H}$ ), 3.28-3.18 (m, 1H), $2.91(\mathrm{~s}, 3 \mathrm{H}), 2.11$ (ddd, $J=12.3,6.9$ and $2.2 \mathrm{~Hz}, 1 \mathrm{H}), 1.92-1.80(\mathrm{~m}, 3 \mathrm{H}), 1.77-1.62(\mathrm{~m}, 6 \mathrm{H}), 1.60-1.56(\mathrm{~m}, 1 \mathrm{H}), 1.57(\mathrm{~s}, 3 \mathrm{H}), 1.55(\mathrm{~s}, 3 \mathrm{H})$, 
1.50-1.42 (m, 2H), $1.33(\mathrm{~d}, J=6.7 \mathrm{~Hz}, 3 \mathrm{H}), 1.30-1.21(\mathrm{~m}, 4 \mathrm{H}), 1.17(\mathrm{~s}, 9 \mathrm{H}), 1.11-1.00(\mathrm{~m}, 2 \mathrm{H})$, $0.79(\mathrm{~d}, J=7.2 \mathrm{~Hz}, 3 \mathrm{H}) ;{ }^{13} \mathrm{C}$ NMR $\left(125 \mathrm{MHz}, \mathrm{C}_{6} \mathrm{D}_{6}\right) \delta 208.7,177.6,138.3,134.2,129.0,116.7$, $100.1,94.3,76.2,75.3,74.5,74.1,73.2,72.5,66.1,62.9,61.6,55.3,47.3,43.6,41.5,40.4,38.7$, $36.2,35.0,34.0,32.4,32.0,27.3,26.5,25.5,24.0,23.6,16.9,16.0$; high resolution mass spectrum (ESI) m/z 687.4076 [(M+Na) $)^{+}$; calcd for $\mathrm{C}_{37} \mathrm{H}_{60} \mathrm{O}_{10} \mathrm{Na}$ : 687.4084].

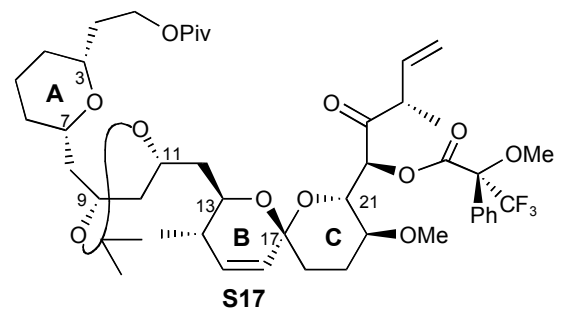

(S)-MTPA ester S17. To a solution of $(+)-\mathbf{S} 16(1.0 \mathrm{mg}, 1.5 \mu \mathrm{mol})$ in $\mathrm{CH}_{2} \mathrm{Cl}_{2}(0.3 \mathrm{~mL})$ at ambient temperature was added DMAP (8 $\mathrm{mg}, 0.065 \mathrm{mmol}, 43$ equiv) and then $(R)-(-)$-MTPA chloride (5 $\mu \mathrm{L}, 0.027 \mathrm{mmol}, 18$ equiv). The resulting mixture was stirred for $15 \mathrm{~min}$. Without work-up, purification by preparative-TLC (3/1, hexanes/EtOAc, $500 \mu \mathrm{m}$ plate) provided (S)-MTPA ester S17 $\left(0.9 \mathrm{mg}, 1 \mu \mathrm{mol}, 70 \%\right.$ yield) as a colorless oil: $\mathrm{R}_{\mathrm{f}} 0.60$ (hexanes/ethyl acetate $\left.3 / 1\right) ;{ }^{1} \mathrm{H}$ NMR $\left(500 \mathrm{MHz}, \mathrm{CDCl}_{3}\right) \delta 7.78(\mathrm{~m}, 2 \mathrm{H}), 7.44-7.40(\mathrm{~m}, 3 \mathrm{H}), 5.84(\mathrm{ddd}, J=17.8,10.1$ and $7.8 \mathrm{~Hz}, 1 \mathrm{H})$, $5.68(\mathrm{~d}, J=2.4 \mathrm{~Hz}, 1 \mathrm{H}), 5.62(\mathrm{dd}, J=10.0$ and $1.8 \mathrm{~Hz}, 1 \mathrm{H}), 5.29(\mathrm{dd}, J=9.9$ and $2.7 \mathrm{~Hz}, 1 \mathrm{H})$, $5.27(\mathrm{~d}, J=17.2 \mathrm{~Hz}, 1 \mathrm{H}), 5.20$ (d, J = 10.2 Hz, 1H), 4.26-4.18 (m, 3H), 4.16-4.09 (m, 1H), 4.06$4.01(\mathrm{~m}, 1 \mathrm{H}), 3.65(\mathrm{~s}, 3 \mathrm{H}), 3.55(\mathrm{app} t \mathrm{~d}, \mathrm{~J}=10.6$ and $5.1 \mathrm{~Hz}, 1 \mathrm{H}), 3.51-3.45(\mathrm{~m}, 2 \mathrm{H}), 3.41-3.36$ (m, 1H), $3.14($ app td, $J=10.6$ and $5.1 \mathrm{~Hz}, 1 \mathrm{H}), 3.07(\mathrm{~s}, 3 \mathrm{H}), 2.06-1.96(\mathrm{~m}, 2 \mathrm{H}), 1.95-1.90(\mathrm{~m}$, 1H), 1.84-1.60 (m, 9H), 1.58-1.48 (m, 6H), $1.38(\mathrm{~s}, 3 \mathrm{H}), 1.36(\mathrm{~s}, 3 \mathrm{H}), 1.32-1.28(\mathrm{~m}, 1 \mathrm{H}), 1.19(\mathrm{~s}$, $9 \mathrm{H}), 1.18(\mathrm{~d}, J=6.9 \mathrm{~Hz}, 3 \mathrm{H}), 0.99(\mathrm{~d}, J=7.2 \mathrm{~Hz}, 3 \mathrm{H})$; high resolution mass spectrum (ESI) $\mathrm{m} / \mathrm{z}$ 903.4495 [(M+Na) ${ }^{+}$; calcd for $\mathrm{C}_{47} \mathrm{H}_{67} \mathrm{O}_{12} \mathrm{~F}_{3} \mathrm{Na:}$ 903.4482]. 


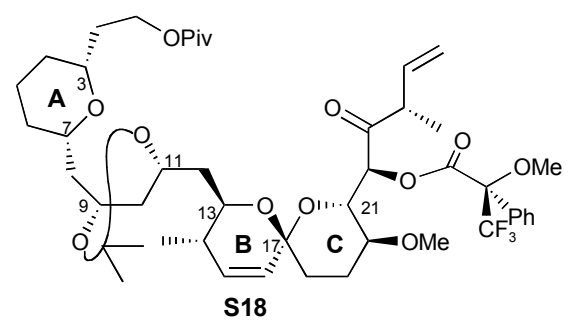

(R)-MTPA ester S18. To a solution of (+)-S16 $(1.0 \mathrm{mg}, 1.5 \mu \mathrm{mol})$ in $\mathrm{CH}_{2} \mathrm{Cl}_{2}(0.4 \mathrm{~mL})$ at ambient temperature was added DMAP ( $8 \mathrm{mg}, 0.065 \mathrm{mmol}, 44$ equiv) and then (S)-(+)-MTPA chloride (5 $\mu \mathrm{L}, 0.027 \mathrm{mmol}, 18$ equiv). The resulting mixture was stirred for $20 \mathrm{~min}$ at ambient temperature. Without work-up, purification by preparative-TLC (4/1, hexanes/EtOAc, $500 \mu \mathrm{m}$ plate) provided (R)-MTPA ester $\mathbf{S} 18\left(1.1 \mathrm{mg}, 1.2 \mu \mathrm{mol}, 80 \%\right.$ yield) as a colorless oil: $\mathrm{R}_{\mathrm{f}} 0.50$ (hexanes/ethyl acetate 3/1); $[\alpha]^{20}{ }_{D}+6.8\left(c 0.10, \mathrm{C}_{6} \mathrm{H}_{6}\right) ;$ IR (film) $3072(\mathrm{w}), 2932$ (s), 2871 (s), $1762(\mathrm{~m}), 1730$ (s),

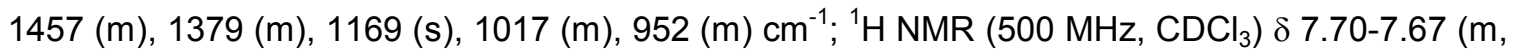
2H), 7.40-7.35 (m, 3H), 5.85 (ddd, $J=17.5,10.3$ and $7.8 \mathrm{~Hz}, 1 \mathrm{H}), 5.72(\mathrm{dd}, J=9.9$ and $1.7 \mathrm{~Hz}$, 1H), $5.62(\mathrm{~d}, J=2.4 \mathrm{~Hz}, 1 \mathrm{H}), 5.48(\mathrm{dd}, J=10.0$ and $2.6 \mathrm{~Hz}, 1 \mathrm{H}), 5.26(\mathrm{~d}, J=17.2 \mathrm{~Hz}, 1 \mathrm{H}), 5.18$ (d, $J=10.2 \mathrm{~Hz}, 1 \mathrm{H}), 4.29$ (dd, $J=9.6$ and $2.5 \mathrm{~Hz}, 1 \mathrm{H}), 4.21$ (app td, $J=10.9$ and $6.6 \mathrm{~Hz}, 1 \mathrm{H}$ ), 4.17-4.11 (m, 2H), 4.08-4.04 (m, 1H), 3.67 (s, 3H), 3.60 (app td, $J=9.3$ and $2.0 \mathrm{~Hz}, 1 \mathrm{H}$ ), 3.51$3.45(\mathrm{~m}, 2 \mathrm{H}), 3.42-3.36(\mathrm{~m}, 1 \mathrm{H}), 3.21$ (app td, $J=10.4$ and $4.9 \mathrm{~Hz}, 1 \mathrm{H}), 3.05(\mathrm{~s}, 3 \mathrm{H}), 2.07-1.95$ (m, 3H), 1.87-1.70 (m, 7H), $1.64(\mathrm{ddd}, J=14.7,9.0$ and $6.0 \mathrm{~Hz}, 1 \mathrm{H}), 1.58-1.48(\mathrm{~m}, 7 \mathrm{H}), 1.39(\mathrm{~s}$, $3 \mathrm{H}), 1.37(\mathrm{~s}, 3 \mathrm{H}), 1.22-1.19(\mathrm{~m}, 1 \mathrm{H}), 1.19(\mathrm{~s}, 9 \mathrm{H}), 1.16(\mathrm{~d}, J=6.7 \mathrm{~Hz}, 3 \mathrm{H}), 1.01(\mathrm{~d}, J=7.2 \mathrm{~Hz}$ $3 \mathrm{H})$; high resolution mass spectrum $(\mathrm{ESI}) \mathrm{m} / \mathrm{z} 903.4504\left[(\mathrm{M}+\mathrm{Na})^{+}\right.$; calcd for $\mathrm{C}_{47} \mathrm{H}_{67} \mathrm{O}_{12} \mathrm{~F}_{3} \mathrm{Na}$ : 903.4482]. 

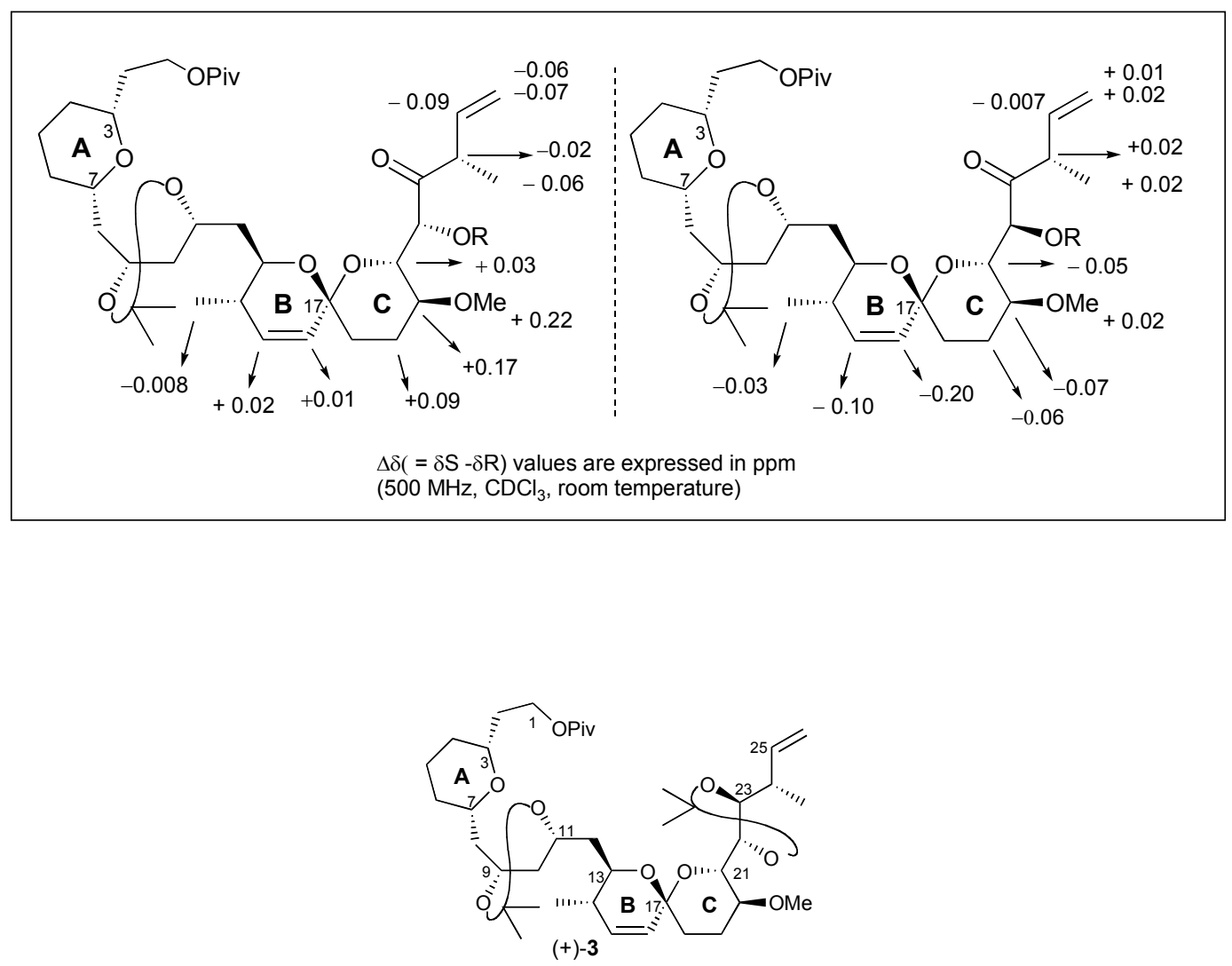

Acetonide (+)-3. To a solution of ketone $(+)-27(1.9 \mathrm{mg}, 2.9 \mu \mathrm{mol})$ in $\mathrm{Et}_{2} \mathrm{O}(0.7 \mathrm{~mL})$ at $0{ }^{\circ} \mathrm{C}$ was added a solution of $\mathrm{Zn}\left(\mathrm{BH}_{4}\right)_{2}\left(0.15 \mathrm{M}\right.$ in $\mathrm{Et}_{2} \mathrm{O}, 0.07 \mathrm{~mL}, 0.011 \mathrm{mmol}, 3.7$ equiv). The resulting solution was stirred for 40 min stirring at $0{ }^{\circ} \mathrm{C}$, and a saturated $\mathrm{NaHCO}_{3}$ aqueous solution $(2 \mathrm{~mL})$ was then added. The mixture was extracted with $\mathrm{Et}_{2} \mathrm{O}(3 \mathrm{~mL} \times 3)$. The combined organic layers were washed with brine $(2 \mathrm{~mL})$, dried over $\mathrm{MgSO}_{4}$, filtered, and concentrated in vacuo. Without further purification, the resulting diol was dissolved in acetone/2,2-dimethoxypropane $(0.7 / 0.1$ $\mathrm{mL}$ ). To the resulting solution at ambient temperature was added, TsOH ( $1 \mathrm{mg}, 5 \mu \mathrm{mol}, 2$ equiv). After 30 min at ambient temperature, triethylamine $(6 \mu \mathrm{L})$ was added, and the resulting mixture was concentrated in vacuo. Flash chromatography on silica gel, using ethyl acetate-hexanes (1:4) as eluant, provided acetonide $(+)-3(1.3 \mathrm{mg}, 1.8 \mu \mathrm{mol}, 64 \%$ yield over 2 steps $)$ as a colorless oil: $\mathrm{R}_{\mathrm{f}} 0.50$ (hexanes/ethyl acetate $\left.3 / 1\right) ;[\alpha]^{20}{ }_{\mathrm{D}}+9.1\left(\mathrm{c} 0.15, \mathrm{C}_{6} \mathrm{H}_{6}\right)$; IR (film) $3078(\mathrm{w})$, 2934 (s), 2872 (s), 1730 (s), 1458 (m), 1377 (m), 1221 (m), 1158 (s), 1103 (s) cm ${ }^{-1} ;{ }^{1} \mathrm{H}$ NMR (500 $\left.\mathrm{MHz}, \mathrm{C}_{6} \mathrm{D}_{6}\right) \delta 6.17(\mathrm{ddd}, J=17.3,10.4$ and $6.7 \mathrm{~Hz}, 1 \mathrm{H}), 5.56(\mathrm{dd}, J=10.0$ and $2.6 \mathrm{~Hz}, 1 \mathrm{H}), 5.44$ (dd, $J=10.0$ and $1.9 \mathrm{~Hz}, 1 \mathrm{H}$ ), 5.11 (app dt, $J=17.3$ and $1.5 \mathrm{~Hz} 1 \mathrm{H}$ ), 5.08 (app dt, $J=10.5$ and 
$1.4 \mathrm{~Hz}, 1 \mathrm{H}), 4.63(\mathrm{~d}, J=6.5 \mathrm{~Hz}, 1 \mathrm{H}), 4.39-4.26(\mathrm{~m}, 4 \mathrm{H}), 3.95(\mathrm{dd}, J=10.0$ and $6.1 \mathrm{~Hz}, 1 \mathrm{H}), 3.93$ (d, $J=9.3 \mathrm{~Hz}, 1 \mathrm{H}$ ), 3.80 (app td, $J=9.5$ and $2.7 \mathrm{~Hz}, 1 \mathrm{H}$ ), 3.61-3.56 (m, 1H), 3.53 (ddd, $J=11.0$, 9.4 and $5.2 \mathrm{~Hz}, 1 \mathrm{H}), 3.31-3.25(\mathrm{~m}, 1 \mathrm{H}), 3.19(\mathrm{~s}, 3 \mathrm{H}), 3.16-3.11(\mathrm{~m}, 1 \mathrm{H}), 2.20$ (ddd, $J=14.3,4.5$ and $2.8 \mathrm{~Hz}, 1 \mathrm{H}), 2.02(\mathrm{ddd}, J=13.0,9.7$ and $6.3 \mathrm{~Hz}, 1 \mathrm{H}), 1.95-1.82(\mathrm{~m}, 4 \mathrm{H}), 1.79-1.72(\mathrm{~m}, 3 \mathrm{H})$, 1.70-1.65 (m, 3H), $1.64(\mathrm{~s}, 3 \mathrm{H}), 1.56(\mathrm{~s}, 3 \mathrm{H}), 1.55$ (s, 3H), 1.47 (app td, J = 13.5 and $4.1 \mathrm{~Hz}, 1 \mathrm{H})$, $1.40(\mathrm{~s}, 3 \mathrm{H}), 1.36-1.22(\mathrm{~m}, 4 \mathrm{H}), 1.20(\mathrm{~d}, \mathrm{~J}=6.7 \mathrm{~Hz}, 3 \mathrm{H}), 1.17(\mathrm{~s}, 9 \mathrm{H}), 1.10-1.02(\mathrm{~m}, 2 \mathrm{H}), 0.76$ (d, $J=7.1 \mathrm{~Hz}, 3 \mathrm{H}) ;{ }^{13} \mathrm{C}$ NMR $\left(125 \mathrm{MHz}, \mathrm{C}_{6} \mathrm{D}_{6}\right) \delta 177.6,142.3,133.5,129.1,113.5,108.5,100.2$, $93.5,81.6,75.8,74.6,74.5,74.1,73.4,72.1,66.3,62.9,61.6,56.1,43.6,42.0,41.2,38.7,37.2$, $36.2,35.1,34.4,32.3,32.0,27.3,27.0,26.5,26.3,25.6,24.0,23.4,17.3,17.2$; high resolution mass spectrum (ESI) m/z $729.4539\left[(\mathrm{M}+\mathrm{Na})^{+}\right.$; calcd for $\mathrm{C}_{40} \mathrm{H}_{66} \mathrm{O}_{10} \mathrm{Na:}$ 729.4554]. 